\title{
SOME RESULTS AND EXAMPLES ABOUT THE BEHAVIOR OF HARMONIC FUNCTIONS AND GREEN'S FUNCTIONS WITH RESPECT TO SECOND ORDER ELLIPTIC OPERATORS
}

\author{
ALANO ANCONA \\ Dedicated to Professor Masayuki Itô on the occasion of \\ his sixtieth birthday
}

\begin{abstract}
Let $M$ be a manifold and let $\mathcal{L}$ be a sufficiently smooth second order elliptic operator in $M$ such that $(M, \mathcal{L})$ is a transient pair. It is first shown that if $\mathcal{L}$ is symmetric with respect to some density in $M$, there exists a positive $\mathcal{L}$-harmonic function in $M$ which dominates $\mathcal{L}$-Green's function at infinity. Other classes of elliptic operators are investigated and examples are constructed showing that this property may fail if the symmetry assumption is removed. Another part of the paper deals with the existence of critical points for certain $\mathcal{L}$-harmonic functions with periodicity properties. A class of small perturbations of second order elliptic operators is also described.
\end{abstract}

\section{§0. Introduction}

In this paper, we consider three questions about the Potential theory with respect to second order elliptic operators. These questions are essentially different in character and apart from the introduction the exposition of the related results will be kept independent.

The first question is due to Y. Pinchover ([Pi2], see also [Pi3], [Pi4] where this property appears). Let $\Omega$ be a domain in $\mathbb{R}^{d}$ (or a Riemannian manifold), let $\mathcal{L}$ be a locally well-behaved second order elliptic operator in $\Omega$ which admits a (non-negative) Green's function in $\Omega$ and let $g$ denote the $\mathcal{L}$-Green's function with respect to $\Omega$ and some given pole $P \in \Omega$. It is then asked if there necessarily exists an $\mathcal{L}$-harmonic function $u$ in $\Omega$ which dominates $g$ in the sense that $g(m)=o(u(m))$ when $m$ tends to the point

Received January 6, 2000.

2000 Mathematics Subject Classification: 31C12, 31C35 60J10, 60J60. 
at infinity in $\Omega$. It will be shown that if $\mathcal{L}$ is symmetric with respect to some smooth density in $\Omega$ (or more generally if $\mathcal{L}$ is quasi-symmetric in an appropriate sense), then Pinchover's question admits a positive answer. See Sections 1-2. In Section 5, using some ancillary results from Sections 3-4, -in particular the triviality of the Martin boundary of some perturbations of the Laplacian in $\mathbb{R}^{d}, d \geq 3$, (Proposition 3.1)-, we construct an example -with $\Omega=\mathbb{R}^{d}, \mathcal{L}=\Delta+V . \nabla$. where $V$ is a smooth vector field in $\mathbb{R}^{d}$ - which shows that the answer is no in general. There are even counter-examples with a Riemannian manifold $M$ of bounded geometry and an operator in the form $\mathcal{L}=\Delta_{M}+V . \nabla$, where $\Delta_{M}$ is the Laplace-Beltrami operator in $M$ and $V$ is a bounded drift (See Section 10). However, in the hyperbolic setting of [An2] and with $\mathcal{L}$ in one of the classes of weakly coercive elliptic operators considered therein, the answer is again yes, in spite of the apparent lack of symmetry (Proposition 1.3).

In Sections 6-8, we consider a question raised by G. Benarous and H. Owhadi about the behavior of certain harmonic functions with respect to an elliptic operator when the operator and the function satisfy some periodicity conditions. An equivalent form of their question asks whether a smooth function $u$ in $T^{d-1} \times \mathbb{R}$ (where $T=\mathbb{R} / \mathbb{Z}$ is the torus) such that $u(x, t+1)=u(x, t)+1$ and such that $\Delta u-\nabla \varphi \cdot \nabla u=0$ for some smooth function $\varphi$ on $T^{d}$ may admit a critical point. It will be shown that the answer is no if $d=2$ (see Section 7) but that there are examples with critical points when $d \geq 3$ (see Section 8). Perhaps the method could be useful in constructing other counter-examples involving critical points.

Finally we mention in Section 9 a stability property of Green's function with respect to some perturbations of elliptic operators (Theorem 5) which is shown to follow from [An4]. In the form of Corollary 9.1, this was conjectured by Y. Pinchover (in a slightly different form) ([Pi2], see also $[\mathrm{Pi} 3])$.

\section{$\S 1$. Domination of Green's function by harmonic functions: state- ment of the results}

Let $M$ be a connected non compact Riemannian manifold and let $\mathcal{L}$ denote a second order (strictly) elliptic operator in $M$. We assume that $\mathcal{L}$ is in divergence form $\mathcal{L}=\operatorname{div}(\mathcal{A}(\nabla \bullet))+D . \nabla \bullet+\operatorname{div}\left(\bullet D^{\prime}\right)+\gamma \bullet$ where $\mathcal{A}$ is a bounded Borel measurable section of the bundle $\operatorname{End}(T(M))$ which is locally uniformly accretive, $D$ and $D^{\prime}$ are Borel measurable vector fields in 
$M$ of class $L_{\mathrm{loc}}^{p}$ and $\gamma$ is a Borel measurable function in $M$ of class $L_{\mathrm{loc}}^{p / 2}$ for some $p>\operatorname{dim}(M)$. It will be clear from the exposition that Theorem 1 below extends to other standard classes of second order elliptic operators (provided that the symmetry assumption in Theorem 1 is satisfied).

Associated to $(M, \mathcal{L})$ there is a well defined local Potential theory (ref. [Sta], [H-H], [Bre], [Her]; see also [An2], [An3] and references therein) and we shall assume that the reader is familiar with the basic related notions. Recall that the pair $(M, \mathcal{L})$ is said to be of the transient type if the cone of positive $\mathcal{L}$-supersolutions is neither empty nor one-dimensional. Equivalently, there exists a Green's function $G(x, y)$ with respect to $\mathcal{L}$ over $M$, that is: for each $y \in M, G_{y}: x \mapsto G(x, y)$ is a positive $\mathcal{L}$-superharmonic function such that: (i) $\mathcal{L}\left(G_{y}\right)=-\delta_{y}$ in the weak sense (and w.r. to the Riemannian measure $\sigma$ in $M$ ) where $\delta_{y}$ is the Dirac measure at $y$, (ii) the function $G_{y}$ has no positive $\mathcal{L}$-harmonic minorant.

We say that $\mathcal{L}$ is quasi-symmetric if moreover for some reference point $O \in M$, the related Naïm kernel $\theta(x, y)=G(x, y) / G(x, O) G(O, y)$ satisfies: $\theta(x, y) \leq C \theta(y, x)$ for all pair $(x, y)$ in $(M \backslash\{O\})^{2}$ and some $C \geq 1$. It is easy to see that this property is then valid with respect to any reference point with the constant $C^{3}$ instead of $C$. This property is also invariant under smooth changes of metric or relativisations (but we do not need these facts) and it obviously implies the quasi-symmetry of the formal adjoint $\mathcal{L}^{*}$ of $\mathcal{L}$. (See Remarks 1.2 below.)

THEOREM 1. Assume that the pair $(M, \mathcal{L})$ is transient and that $\mathcal{L}$ is quasi-symmetric. Then, there exists a positive $\mathcal{L}$-harmonic function $u$ in $M$ such that, for each $y \in M$,

$$
\lim _{x \rightarrow \infty_{M}} \frac{G(x, y)}{u(x)}=0 .
$$

We have denoted $\infty_{M}$ the point at infinity of $M$. Notice that the completeness of $M$ is not required.

Remark 1.1. (i) The proof will show that $u$ may be taken as the sum of a series of minimal $\mathcal{L}$-harmonic functions. (ii) Note that even if $\mathcal{L}$ is Markovian (i.e. constants are $\mathcal{L}$-harmonic) it is not necessarily the case that $\lim _{x \rightarrow \infty_{M}} G\left(x, x_{0}\right)=0$ for some $x_{0} \in M$.

${ }^{*}$ Every $\mathcal{L}$-superharmonic function $u \not \equiv \infty$ is in $H_{\mathrm{loc}}^{1, q}(M)$ for all $q<N /(N-1), N=$ $\operatorname{dim}(M)$, and " $\mu=-\mathcal{L}(u)$ in the weak sense" defines a positive Radon measure $\mu$ in $M$. See [Sta], [H-H]. 
Remarks 1.2. Assume $\mathcal{L}$ is transient and (for simplicity of proof and statement) sufficiently smooth, say $\mathcal{A}$ is $C^{1}, D$ and $\gamma$ are locally bounded and $D^{\prime}=0$. Fix also a reference point $O \in M$. A. The following properties are equivalent: (a) There is a measurable function $f$, positive and locally bounded in $M$, such that $\int \mathcal{L}(u) v f d \sigma=\int u \mathcal{L}(v) f d \sigma$ for $u, v$ in $C_{0}^{\infty}(M)$, (b) $(x, y) \mapsto G(x, y) h(x)$ is symmetric for some positive function $h$ in $M$, (c) the Naïm kernel is symmetric. Moreover $f$ in (a) and $h$ in (b) must be proportional. B. If $\mathcal{L} \equiv \Delta_{M} \bullet+D . \nabla \bullet+\gamma \bullet$, the Naïm kernel is symmetric iff $D=\nabla \varphi$ for some $\varphi \in C^{0,1}(M)$.

Remarks 1.2 are proved in Section 11. It should be stressed here that if $\mathcal{L}$ is a second order elliptic operator in $\mathbb{R}^{d}$, say in divergence form, locally uniformly elliptic, with locally bounded coefficients and such that $\mathcal{L}(1) \leq 0$, and if we take for $M$ a bounded region in $\mathbb{R}^{d}$, then Theorem 1 follows quickly from the fact that the set of Dirichlet-irregular boundary points is polar. Theorem 1 and the next Theorem 2 deal with the case where $\Omega$ is unbounded, or stated differently, with the case where $\mathcal{L}$ degenerates near the boundary.

In contrast with Theorem 1, we shall see that for general non self-adjoint elliptic operators its conclusion may fail even when the Martin boundary is reduced to a single point.

THEOREM 2. For each $d \geq 3$ there exists a smooth vector field $V$ in $\mathbb{R}^{d}$ such that for $\mathcal{L}=\Delta \bullet+V . \nabla \bullet$, the pair $\left(\mathbb{R}^{d}, \mathcal{L}\right)$ is transient, positive $\mathcal{L}$-harmonic functions are constant, but the $\mathcal{L}$-Green function with pole at some $P \in M$ does not vanish at infinity.

We have denoted above $\Delta$ the Laplace operator in $\mathbb{R}^{d}$. A proof of Theorem 2 is given in Section 5 after some preliminaries in Sections 3 and 4 . Theorem 2 extends to the case $d=2$ (see 5.7) but our proof of this involves extra technicalities and details will be omitted here.

A variant of the construction leads also to the following.

Theorem $2^{\prime}$. There exists a complete Riemannian manifold $M$ of $d i$ mension 2 , with bounded curvatures and injectivity radius bounded from below, a smooth bounded vector field $V$ in $M$ such that for $\mathcal{L}=\Delta_{M} \bullet+V$. $\bullet \bullet$, the pair $(M, \mathcal{L})$ is transient, positive $\mathcal{L}$-harmonic functions are constant, but the $\mathcal{L}$-Green function with pole at some $P \in M$ does not vanish at infinity. 
The proof of Theorem $2^{\prime}$ (and of its discrete version) is sketched at the end in Section 10.

However, there are classes of operators $\mathcal{L}$ over complete Riemannian manifolds which might seem far from formally self-adjoint but to which Theorem 1 applies. We shall see this for the divergence type elliptic operators considered in [An2] as an application of Theorem 1.

Assume now that $M$ is complete and that the coefficients of $\mathcal{L}$ are such that for some $p>\operatorname{dim}(M)$ and some $\theta>1$,

$$
\begin{gathered}
\theta^{-1}|\xi|^{2} \leq\left\langle\mathcal{A}_{a}(\xi), \xi\right\rangle \leq \theta|\xi|^{2}, \\
\left\|\mathcal{A}_{a}\right\|_{\operatorname{End}\left(T_{a}(M)\right)}+\|D\|_{L^{p}\left(B_{a}\right)}+\left\|D^{\prime}\right\|_{L^{p}\left(B_{a}\right)}+\|\gamma\|_{L^{p / 2}\left(B_{a}\right)} \leq \theta
\end{gathered}
$$

when $a \in M$ and $\xi \in T_{a}(M)$ and where $B_{a}=B(a, 1)$.

Proposition 1.3. Assume moreover that $M$ is a Cartan-Hadamard manifold with pinched negative sectional curvatures and that $\mathcal{L}$ is weakly coercive. Then, $\mathcal{L}$ is quasi-symmetric.

Recall that $\mathcal{L}$ is called weakly coercive if $\mathcal{L}+\varepsilon I$ admits a positive supersolution for some $\varepsilon>0$. This implies the existence of a Green's function for $\mathcal{L}$.

Remark 1.4. The proof (see Section 2.D) is easily extended to the case of a Gromov hyperbolic manifold $M$ with bounded geometry (See [An3]). It extends also to the classes of non divergence type elliptic operators considered in [An2].

ExAmPle 1.5. 1. Proposition 1.3 applies to every operator $\mathcal{L}=\Delta+$ $D . \nabla$ with $D$ a Borel vector field in the hyperbolic space $M=H_{N}(-1)$ if $|D| \leq C_{0}, C_{0}<N-1$. (Check the weak coercivity by using $u(x)=x_{N}^{\beta}$ in the half-space model $M=\left\{x \in \mathbb{R}^{N} ; x_{N}>0\right\}$ of $H_{N}(-1)$ with $\beta>0$ small.) By Remarks 1.2, the corresponding Naïm kernel is quasi-symmetric but not symmetric if moreover $D$ is not a gradient. 2. From Proposition 1.3, various classes of elliptic operators over regions in $\mathbb{R}^{d}$ for which Pinchover's question admits a positive answer may be deduced (see [An2], §8). Let us only mention here the case of a bounded Lipschitz domain $\Omega$ (some more general domains are admissible too) equipped with an elliptic operator in the form $L=\operatorname{div}(A \nabla)+.B . \nabla .-\gamma$. with $|B(x)|=o(1 / d(x, \partial \Omega))$ for $d(x, \partial \Omega) \rightarrow 0, \gamma \geq 0,|\gamma(x)| \leq c / d(x, \partial \Omega)^{2}$ and a uniformly bounded and coercive matrix $A(x)$. Proposition 1.3 says that the Naïm kernel of $L$ is quasi-symmetric. 


\section{§2. Proof of Theorem 1 and Proposition 1.3}

The argument will combine an extension to the present framework of results of L. Naïm [Naï] with some abstract Potential theoretic results about extended versions of Fekete's transfinite diameter (ref. [Ev], [Cho], [Ni]). For the needed facts from Martin's boundary theory, we refer the reader to [An3] and references there.

We fix a point $O \in M$ and denote $K_{x}(y)=G(y, x) / G(O, x), y \in M$, $x \in M \backslash\{O\}$, the related $\mathcal{L}$ Martin kernel. In general a star in the notations to follow refers to the adjoint $\mathcal{L}^{*}$ of $\mathcal{L}$. So $K_{x}^{*}(y)=G(x, y) / G(x, O), x \in$ $M \backslash\{O\}, y \in M$, is the adjoint Martin kernel. As before, we set $\theta(x, y)=$ $G(x, y) / G(x, O) G(O, y)$ for $x, y$ in $M \backslash\{O\}$.

\section{A.}

We require the compactification of $M$ (i.e. a compact topological space containing $M$ as a dense open subspace) which is in some sense the smallest containing both the $\mathcal{L}$ Martin compactification and the $\mathcal{L}^{*}$ Martin compactification of $M$. This is the (unique up to equivalence) metrizable compactification $\bar{M}=M \cup \partial M$ of $M$ having the following property: if $\left\{x_{n}\right\}$ is a sequence converging to the point at infinity in $M$, then $\left\{x_{n}\right\}$ converges to some point $\zeta$ in $\partial M$ if and only if both sequences $\left\{K_{x_{n}}\right\}$ and $\left\{K_{x_{n}}^{*}\right\}$ converge pointwise in $M$. If $\zeta \in \partial M$ is the limit point in $\bar{M}$ of such a sequence $\left\{x_{n}\right\}$, we define $K_{\zeta}=\lim _{n \rightarrow \infty} K_{x_{n}}$ and $K_{\zeta}^{*}=\lim _{n \rightarrow \infty} K_{x_{n}}^{*}$. (Of course $K_{\zeta}$ and $K_{\zeta}^{*}$ depend only on $\zeta$ not on the specific sequence.)

Clearly $\partial M$ projects on each of the Martin's boundaries $\widehat{\partial} M$ and $\widehat{\partial}^{*} M$ corresponding to $\mathcal{L}$ and $\mathcal{L}^{*}$. Moreover if we restrict to the minimal boundaries we have natural one to one maps.

Lemma 2.1. Let $\zeta \in \partial M$. The kernel function $K_{\zeta}$ is a minimal positive $\mathcal{L}$ harmonic function if and only if $K_{\zeta}^{*}$ is $\mathcal{L}^{*}$ minimal. Moreover if $K_{\zeta}$ is $\mathcal{L}$ minimal, then a sequence $\left\{x_{n}\right\}$ in $M$ converges to $\zeta$ in $\bar{M}$ iff $\left\{K_{x_{n}}\right\}$ converges pointwise to $K_{\zeta}$.

Proof. Set $\Phi(O)=1$ and $\Phi(x)=G(O, x) / G(x, O)$, for $x \in M \backslash\{O\}$. For every $\zeta \in \bar{M} \backslash\{O\}$, we have by the quasi-symmetry assumption

$$
C^{-1} \Phi(x) K_{\zeta}(x) \leq K_{\zeta}^{*}(x) \leq C \Phi(x) K_{\zeta}(x), \quad x \in M
$$

where $C$ is a quasi symmetry constant for $\mathcal{L}$. Using Martin's integral representation of positive $\mathcal{L}$ (or $\mathcal{L}^{*}$ ) harmonic functions, it follows that for 
each non-negative $\mathcal{L}$ harmonic function $h$ in $M$ there is a (non-unique) $\mathcal{L}^{*}$ harmonic function $h^{*}$ such that

$$
C^{-1} \Phi(x) h(x) \leq h^{*}(x) \leq C \Phi(x) h(x)
$$

in $M$ (and vice-versa). From these facts the first claim follows immediately.

The second claim is then easily checked: if $K_{x_{n}} \rightarrow K_{\zeta}$ and if $K_{\zeta}$ is $\mathcal{L}$-minimal, then by (2.1) any function $h^{*}$ which is a limit of a subsequence of $\left\{K_{x_{n}}^{*}\right\}$, must satisfy $C^{-2} K_{\zeta}^{*} \leq h^{*} \leq C^{2} K_{\zeta}^{*}$ and since $K_{\zeta}^{*}$ is minimal and $h(O)=1$, we have $h^{*}=K_{\zeta}^{*}$.

In other words if we set $\partial_{1} M=\left\{\zeta \in \partial M ; K_{\zeta}\right.$ is $\mathcal{L}$ minimal $\}, M \cup$ $\partial_{1} M$ (as a subspace of $\bar{M}$ ) is homeomorphic to the minimal Martin space $M \cup \widehat{\partial}_{1} M$ (as a subspace of the $\mathcal{L}$ Martin's compactification $\widehat{M}_{\mathcal{L}}$ ) and also to $M \cup \widehat{\partial}_{1}^{*} M$ (as a subspace of $\widehat{M}_{\mathcal{L}^{*}}$ ). We collect two other easy facts.

Lemma 2.2. Let $\zeta \in \partial_{1} M$ and let $A \subset M$. The set $A$ is minimally thin at $\zeta$ with respect to $\mathcal{L}$ iff it is minimally thin at $\zeta$ with respect to $\mathcal{L}^{*}$.

Proof. We may assume that $O \notin \bar{A}$. Recall that $A$ is thin at $\zeta_{0}$, if the réduite function $\widehat{R}_{K_{\zeta_{0}}}^{A}$ is a $\mathcal{L}$-potential. That is, there exists a nonnegative measure $\mu$ in $M$ such that $O \notin \operatorname{supp}(\mu)$ and: (i) $G \mu(O)$ is finite (ii) $G \mu \geq K_{\zeta_{0}}$ in $A$. Setting $\nu(d Y)=G(O, Y) \mu(d Y)$, it is seen that $A$ is thin at $\zeta_{0}$ if and only if there exists a non-negative Borel measure $\nu$ in $M$ such that $O \notin \operatorname{supp}(\nu), \theta_{\nu} \geq \theta\left(., \zeta_{0}\right)$ in $A$ and $\theta_{\nu} \not \equiv \infty$ (i.e. $\left.\nu(M)<\infty\right)$. Here we have let $\theta_{\nu}=\int_{M \backslash\{O\}} \theta(., y) d \nu(y)$ and $\theta\left(., \zeta_{0}\right)=\lim _{y \rightarrow \zeta_{0}} \theta(., y)=K_{\zeta_{0}}(.) / G(., O)$. Applying this also to $\mathcal{L}^{*}$, the lemma follows from the quasi-symmetry.

Let $\eta \in \partial M$ and let $\lambda_{\eta}$ (resp. $\lambda_{\eta}^{*}$ ) denote the Martin's representing measure of $K_{\eta}$ (resp. $K_{\eta}^{*}$ ) on $\partial_{1} M$, i.e. $K_{\eta}()=.\int_{\partial_{1} M} K_{\zeta}(.) d \lambda_{\eta}(\zeta)$ and $K_{\eta}^{*}()=.\int_{\partial_{1} M} K_{\zeta}^{*}(.) d \lambda_{\eta}^{*}(\zeta)$.

LEMma 2.3. We have $C^{-1} \lambda_{\eta} \leq \lambda_{\eta}^{*} \leq C \lambda_{\eta}$ for some constant $C \geq 1$.

Proof. This is a consequence of the uniqueness of Martin's integral representation by minimal functions, together with Lemma 2.1 and (2.1), (2.2). 


\section{B.}

We may now define the Naïm kernel for our setting. Simple adaptations of the arguments in [Naï] shows the following. Let $\left\{L_{j}\right\}$ be an exhaustion of $M$ by an increasing sequence of relatively compact regions containing $O$. For $j \geq 1$ and $\eta \in \bar{M} \backslash\{O\}$, let $\nu_{j}^{\eta}$ (resp. $\nu_{j}^{* \eta}$ ) denote the positive measure in $M$ such that $G \nu_{j}^{\eta}=R_{K_{\eta}}^{L_{j}}$ (resp. $\left.G^{*}\left(\nu_{j}^{* \eta}\right)={ }^{*} R_{K_{\eta}^{*}}^{L_{j}}\right)$. We may then set, for $(\zeta, \eta) \in(\bar{M} \backslash\{O\})^{2}:$

$$
\theta(\zeta, \eta)=\sup _{j \geq 1} \int K_{\zeta}^{*}(z) d \nu_{j}^{\eta}(z)=\sup _{j \geq 1} \int K_{\eta}(z) d \nu_{j}^{* \zeta}(z) .
$$

This defines a positive l.s.c. kernel $\theta$ in $\bar{M} \backslash\{O\}$ such that $\theta(., \eta)=$ $K_{\eta}(.) / G(., O)$ in $M \backslash\{O\}, \theta(\zeta,)=.K_{\zeta}^{*}(.) / G(O,$.$) in M \backslash\{O\}$. Moreover, for every positive measure $\mu$ in $\bar{M} \backslash\{O\}$ and every $\zeta \in \partial_{1} M$, the $\theta$ potential $\theta_{\mu}()=.\int \theta(., \eta) d \mu(\eta)$ satisfies:

$$
\theta_{\mu}(\zeta)=\liminf _{x \in M, x \rightarrow \zeta} \theta_{\mu}(x)=f^{*} \lim _{x \rightarrow \zeta} \theta_{\mu}(x)=f \lim _{x \rightarrow \zeta} \theta_{\mu}(x)
$$

where $f \lim _{x \rightarrow \zeta}$ (resp. $f^{*} \lim _{x \rightarrow \zeta}$ ) denotes a fine limit at $\zeta$ w.r. to $\mathcal{L}$ (resp. w.r. to $\mathcal{L}^{*}$ ). (See [Naï, pp. 226-227] for the first two equalities and note that the third follows by Lemma 2.2.)

It follows also easily from the definitions that for $x$ and $y$ in $\partial M,-$ recall $\lambda_{x}^{*}$ (resp. $\lambda_{y}$ ) is the adjoint representing measure of $x$ in $\partial_{1} M$ (resp. the representing measure of $y$ in $\left.\partial_{1} M\right)$

$$
\theta(x, y)=\int \theta(\zeta, y) d \lambda_{x}^{*}(\zeta)=\int \theta(x, \eta) d \lambda_{y}(\eta)
$$

In particular, the following holds.

LEMMA 2.4. The kernel $\theta$ is quasi-symmetric in $\partial M \times \partial M$ (i.e. $\theta(\zeta, \eta) \geq C \theta(\eta, \zeta)$ for some constant $C \geq 1)$. The $\theta$-energy $\theta(\mu, \mu)=$ $\iint \theta(\zeta, \eta) d \mu(\zeta) d \mu(\eta)$ of every probability measure $\mu$ in $\partial M$ is infinite.

Proof. The first claim follows from (2.3) (and its adjoint version), (2.4) and Lemma 2.3. By (2.4) and Fubini, we have the identity $\theta(\mu, \mu)=$ $\theta\left(\nu^{\prime}, \nu^{\prime \prime}\right)$ where $\nu^{\prime}=\int \lambda_{x} d \mu(x), \nu^{\prime \prime}=\int \lambda_{x}^{*} d \mu(x)$. By the general FatouDoob-Naïm theorem and (2.3), we have that for every positive measure $\nu$ in $\partial M, \theta_{\nu}(\zeta)=+\infty$ at $\nu$ a.a. $\zeta$ in $\partial_{1} M$. Using Lemma 2.3 again, the result follows.

In particular $\theta$ is infinite on the diagonal of $(\bar{M} \backslash\{O\})^{2}$. 


\section{C. Conclusion}

We are now in good position to apply the results in [Cho] to the symmetric kernel $N(x, y)=\theta(x, y)+\theta(y, x)$ over the compact space $\partial M$. Following [Cho], define the Fekete constant $\delta(A)$ of the set $A \subset \partial M$ (w.r. to the kernel $N$ ) as follows. For each finite subset $X$ of $A$ consisting of $n$ points, set $N(X)=\frac{1}{n(n-1)} \sum_{x, y \in X^{2}, x \neq y} N(x, y)$ and let $\delta_{n}(A)=\inf \{N(X) ;|X|=$ $n, X \subset A\}$. The Fekete constant of $A$ is then $\delta(A)=\sup _{n \geq 1} \delta_{n}(A)$. So $\delta(A)=+\infty$ if $A$ is finite.

Now $N$ is a non-negative lower semicontinuous kernel on the compact space $\partial M$ which is infinite on the diagonal. Moreover, the $N$-energy of every probability measure $\mu$ on $\partial M$ is infinite. It follows then that $\delta(\partial M)=+\infty$ (i.e. $\partial M$ has zero $N$-transfinite diameter) -see Lemme 2 in [Cho]-. Next, Proposition 1 in [Cho] ensures the existence of a probability measure $\mu$ on $\widehat{\partial} M$ of the form $\mu=\sum_{n>1} \alpha_{n} \delta_{\zeta_{n}}$-where $\alpha_{n} \geq 0$, and $\delta_{\zeta_{n}}$ is Dirac measure at $\zeta_{n}-$ such that $N_{\mu}(\zeta)=+\infty$ for every $\zeta \in \partial M$, which means that $\theta_{\mu}=\infty$ in $\partial M$. Since $\theta_{\mu}$ is lower semi-continuous, we must then have $\lim _{y \in M, y \rightarrow \zeta}\left[K_{\mu}(y) / G(O, y)\right]=+\infty$ for all $\zeta \in \partial M$. In other words, $\lim _{y \rightarrow \infty_{M}}\left[K_{\mu}(y) / G(O, y)\right]=+\infty$ and Theorem 1 is proven.

\section{D. Proof of Remark 1.1 (i)}

We have seen that $\delta(\partial M)=\infty$ and since $\partial_{1} M \subset \partial M$ it follows that $\delta\left(\partial_{1} M\right)=+\infty$. By Proposition 1 from [Cho] (where no topological assumption is made on the set $A$ ) there is a probability measure $\mu=\sum_{n=1}^{\infty} \alpha_{n} \delta_{\zeta_{n}}$ with $\zeta_{n} \in \partial_{1} M$ and $\alpha_{n} \geq 0$ such that $N_{\mu}(\zeta)=+\infty$ for $\zeta \in \widehat{\partial}_{1} M$. It follows by $(2.4)$ that $\theta_{\mu}=+\infty$ on $\partial M$. Whence the remark by the end of the proof above.

\section{E. Proof of Proposition 1.3}

The main point in the assumptions of Proposition 1.3 is that they imply the following boundary Harnack inequalities (ref. [An2], [An3]):

$$
C^{-1} G(X, Y) G(Y, Z) \leq G(X, Z) \leq C G(X, Y) G(Y, Z)
$$

for all $X, Y, Z$ in $M$ with $d(X, Y) \geq 1, d(Y, Z) \geq 1$ and $Y$ in the geodesic segment $X Z$. Here $C$ is a constant depending only on $\mathcal{L}$. Recall also that (for another constant $C=C(\mathcal{L})) C^{-1} \leq G(X, Y) \leq C$ for all $X, Y$ in $M$ with $d(X, Y)=1$. By standard local results for second order elliptic operators it follows that

$$
C^{-1} \varphi(d(X, Y)) \leq G(X, Y) \leq C \varphi(d(X, Y))
$$


for $d(X, Y) \leq 10$ where $\varphi(t)=t^{2-N}$ if $N=\operatorname{dim}(M) \geq 3$ and $\varphi(t)=$ $1+\log (10 / t)$ if $N=2$.

By Harnack inequalities and (2.6), to prove the quasi-symmetry of $\theta$ it is enough to consider the case where $X \in M \backslash B(O, 4), Y \in M \backslash B(O, 4)$ and $d(X, Y) \geq 4$.

Let $H$ denote the point nearest to $O$ on the geodesic segment $X Y$ with the convention that if this minimizing point is in $B(O, 1)$ (resp. in $B(X, 1)$, or in $B(Y, 1)$ ) we take $H=O$ (resp. $H=X, H=Y$ ) instead. By $(2.5)$ and if $H \neq X$ and $H \neq Y$, we have

$$
C^{-1} G(X, H) G(H, Y) \leq G(X, Y) \leq C G(X, H) G(H, Y)
$$

If $H=O$ (i.e. $d(O,[X, Y]) \leq 1)$ this means that $C^{-1} \leq \theta(X, Y) \leq C$ and so $\theta(X, Y) \leq C^{2} \theta(Y, X)$. Assume now $H \neq O$ (and $H \neq X, H \neq Y$ ). By the assumptions on $M$, the distance of $H$ to each geodesic segment $O X$, $O Y$ is bounded by a constant (see e.g. [An3, 2.6, p. 82]). Thus by (2.5) and Harnack inequalities, we also have

$$
C^{-1} G(X, H) G(H, O) \leq G(X, O) \leq C G(X, H) G(H, O)
$$

and similar inequalities for $G(Y, O)$. Whence,

$$
\frac{C^{-3}}{G(O, H) G(H, O)} \leq \theta(X, Y) \leq \frac{C^{3}}{G(O, H) G(H, O)}
$$

Moreover it is easily checked that this holds also if $X=H$ or $Y=H$. The proposition follows.

Remark 2.5. Using (2.7) one may also show that $d_{\theta}(x, y)=\theta(x, y)^{-1}$ satisfies the quasi-metric inequality considered in [K-V] (see Section 7, inequality (7.6) there). A proof of the quasi-metric property conjectured by Kalton-Verbitsky ([K-V, p. 3488, top]) follows. Details and extensions will hopefully appear elsewhere.

We next turn to the proof of Theorem 2. The first step describes a set of perturbations of the Laplacian in $\mathbb{R}^{d}$ having trivial Martin boundaries. 


\section{$\S 3 . \quad$ Triviality of the Martin boundary of some diffusions in $\mathbb{R}^{d}$}

Until the end of Section $5, d$ is a fixed integer $\geq 3$. The origin in $\mathbb{R}^{d}$ is denoted $O$ and a point $x=\left(x_{1}, \ldots, x_{d}\right)$ in $\mathbb{R}^{d}$ will often be written as $\left(x_{1}, x^{\prime}\right)$, with $x^{\prime}=\left(x_{2}, \ldots, x_{d}\right) \in \mathbb{R}^{d-1}$. Denote $\Lambda\left(\mathbb{R}^{d}\right)$ the set of all second order (strictly) elliptic operator $\mathcal{L}$ in $\mathbb{R}^{d}$ with smooth coefficients and such that $\mathcal{L}(1) \leq 0$ and let $\Delta$ denote the standard Laplacian in $\mathbb{R}^{d}$.

In the next statement, we set $A_{n}=\left(-2^{n}, 0, \ldots, 0\right)$.

Proposition 3.1. Let $\Phi \subset \mathbb{R}^{d}$ be a closed subset of the half-space $\left\{\left(x_{1}, \ldots, x_{d}\right) \in \mathbb{R}^{d} ; x_{1} \geq 1\right\}$ which is thin at infinity w.r. to $\Delta$. Let $\mathcal{L} \in$ $\Lambda\left(\mathbb{R}^{d}\right)$ be such that $\mathcal{L}=\Delta$ outside $\Phi$. Assume moreover that there exists a positive constant $c_{1}$ such that for every integer $n \geq 0$, every positive $\mathcal{L}$-harmonic function $u$ in $B\left(0,2^{n+1}\right)$ and every $x \in B\left(O, 2^{n}\right)$, we have

$$
u(x) \leq c_{1} u\left(A_{n}\right) .
$$

Then, $\mathcal{L}$ admits a Green's function in $\mathbb{R}^{d}$ and the Martin boundary of $\mathbb{R}^{d}$ w.r. to $\mathcal{L}$ is reduced to a single point.

Remark 3.2. 1. The proof (or a simple direct argument) shows that the minimal $\mathcal{L}$-harmonic function normalized at $O$ is bounded in $\mathbb{R}^{d}$ and has a positive lower bound in $\left\{\left(t, x^{\prime}\right) ; t \leq 0, x^{\prime}=0\right\}$. 2. There exists a complete Riemannian manifold $M$ of bounded geometry admitting a non trivial Martin boundary and a (minimizing) geodesic ray $\gamma:[0, \infty) \rightarrow M$, such that for some constant $C \geq 1$, one has $u(x) \leq C u(\gamma(t))$ for $t \geq 1, x \in$ $B(\gamma(0), t)$ and $u$ positive harmonic in $B(\gamma(0), 2 t)$. An example is obtained by glueing, along its boundary circle, a flat half cylinder $\mathcal{C}$ to a $\mathbb{Z}^{3}$ cover $N$ of a compact Riemannian surface (after removing a disc in $N$ ) and on taking for $\gamma$ a fixed ray in $\mathcal{C}$.

Proof of Proposition 3.1. The proof is expounded in four simple steps. By adding a ball to $\Phi$, we may assume that $\Phi$ has nonempty interior.

Step 1. The thinness assumption means that the réduite function (ref. [Bre]) $v=\widehat{R}_{1}^{\Phi}$ with respect to $\Delta$ is a $\Delta$ potential (and thus $v \not \equiv 1$ ). Clearly, $v$ is also the réduite $\widehat{R}_{1}^{\Phi}$ w.r. to $\mathcal{L}$ and hence $\mathcal{L}$-superharmonic. Since $v$ and 1 are not proportional, $\left(\mathbb{R}^{d}, \mathcal{L}\right)$ is transient. (If $\mathcal{L}(1) \not \equiv 0$ the transient character of $\mathcal{L}$ is immediate.)

Step 2. We show that there exists a constant $c_{2}>0$ depending on $\mathcal{L}$ and such that for every integer $n \geq 0$ and every positive $\mathcal{L}$-harmonic 
function $u$ in $B_{n+1}:=B\left(O, 2^{n+1}\right)$, we have, if $e_{1}$ denotes the unit vector $(1,0, \ldots, 0)$ in $\mathbb{R}^{d}$,

$$
\min \left\{u\left(-t e_{1}\right) ; 0 \leq t \leq 2^{n}\right\} \geq c_{2} u\left(A_{n}\right)
$$

By Harnack inequalities (for $\Delta$ ) we have $u(x) \geq c u\left(A_{n}\right)$ for $x \in B\left(A_{n}, 2^{n-1}\right.$ ) and some $c=c(d)>0$. On the other hand, $u=u_{1}+u_{2}$ in $U_{n}=B_{n} \backslash \Phi$, where $u_{1}$ (resp. $u_{2}$ ) is the bounded $\Delta$-harmonic function in $B_{n}$ (resp. in $\left.U_{n}\right)$ such that $u_{1}=u$ on $\partial B_{n}$ (resp. $u_{2}=1_{\Phi}\left(u-u_{1}\right)$ on $\left.\partial U_{n}\right)$. By the first sentence of the paragraph and by Poisson integral formula, there is a constant $c^{\prime}=c(d)$ such that $u_{1} \geq c^{\prime} c u\left(A_{n}\right)$ in $B\left(A_{n-1}, 2^{n-1}\right)$ and, by the assumption, $u_{1}(x) \leq c_{1} u\left(A_{n}\right)$ in $B_{n}$. Whence,

$$
u(x) \geq u\left(A_{n}\right)\left(c^{\prime \prime}-c_{1} v(x)\right), \quad x \in B\left(A_{n-1}, 2^{n-1}\right) .
$$

where $c^{\prime \prime}=c^{\prime \prime}(d)>0$ and, as above, $v=\widehat{R}_{1}^{\Phi}$.

Thus, estimate (3.1) with $t$ restricted to $\left[t_{0}, 2^{n}\right]$ holds if $v\left(-t e_{1}\right) \leq$ $c^{\prime \prime} / 2 c_{1}$ for all $t \geq t_{0}$. But this condition holds for $t_{0}$ large enough since $v(O)<\infty$ and $v\left(-t e_{1}\right)=\int_{\Phi}\left|\left(-t e_{1}\right)-y\right|^{-(d-2)} d \mu(y) \rightarrow 0$ for $t \rightarrow+\infty$ by the Lebesgue dominated convergence theorem.

Once we have chosen and fixed $t_{0}$ (depending on $\Phi$ ), the bound $u\left(-t e_{1}\right)$ $\geq c_{2} u\left(A_{n}\right), 0 \leq t \leq 2^{n}$, follows from Harnack inequalities for the compact set $\left\{-t e_{1} ; 0 \leq t \leq t_{0}\right\}$ and the domain $\mathbb{R}^{d} \backslash \Phi$. This proves (3.1).

Step 3. Let $u$ be a positive $\mathcal{L}$-harmonic function in $\mathbb{R}^{d}$. By the above and Harnack inequalities, we have that $u(x) \sim u\left(A_{n}\right) \sim u(O)$ for $x \in D_{n}:=$ $B\left(-2^{n} e_{1}, 2^{n-1}\right)$, where $A \sim B$ means that $\alpha^{-1} \leq A / B \leq \alpha$ for some real $\alpha \geq 1$ depending only on $\mathcal{L}$.

For $n \geq 1$, let $H_{n}$ denote the réduite function (with respect to $\mathcal{L}$ and over $\left.\mathbb{R}^{d}\right) H_{n}=R_{1}^{D_{n}}$ where $D_{n}=B\left(-2^{n} e_{1}, 2^{n-1}\right)$ as before. By the above and by the very definition of $H_{n}$, we have $H_{n} \leq c u / u(O)$ in $\mathbb{R}^{d}$ (where $c$ does not depend on $n$ or on $u$ ). Thus if $H$ is a cluster value of $\left\{H_{n}\right\}$ for $n \rightarrow \infty$, then $H \leq c u / u(0)$ in $\mathbb{R}^{d}$. Notice also that $H \leq 1$.

On the other hand, at $x \in \mathbb{R}^{d} \backslash D_{n}$, the harmonic measure of $D_{n}$ in $\mathbb{R}^{d}$ -w.r. to $\Delta$ - is $\left(2^{n-1} /\left|d\left(x, A_{n}\right)\right|\right)^{d-2}$ and so is larger than $c_{3}=2^{-d+2}$ for $x=$ $-t e_{1}, 0 \leq t \leq 2^{n-1}$. By comparison with the harmonic measure within the region $\mathbb{R}^{\bar{d}} \backslash \Phi$, it follows that for such $t$ we have $H_{n}\left(-t e_{1}\right) \geq c_{3}-v\left(-t e_{1}\right)$, so that for $t$ fixed sufficiently large $H_{n}\left(-t e_{1}\right) \geq c_{3} / 2$ when $n \geq \log (t) / \log (2)$. Thus, $H\left(-t e_{1}\right)>c_{3} / 2$ and the function $H$ is positive. 
Step 4. Conclusion. If $u$ is taken to be minimal in $\mathbb{R}^{d}$ normalized at 0 (by Step 1 there is at least one such $u$ ) $H$ must be proportional to $u$. Hence, $u$ is unique which means that all positive $\mathcal{L}$-harmonic functions are proportional to $H$ and the Martin boundary consists in exactly one point. Remark 3.2.1 follows now from the boundedness of $H$, Harnack inequalities and the estimate from below of $H\left(-t e_{1}\right)$, for $t$ positive and large, in Step 3.

\section{§4. Other preliminaries to the proof of Theorem 2}

\section{1.}

We first recall an estimate of the Harnack-near-the-boundary type which will be used to check that the basic assumption in Proposition 3.1 is fulfilled by our construction.

Proposition 4.1. ([An1]) Let $\Omega$ be a domain in $\mathbb{R}^{d}$ with Green function $G$ (with respect to the Laplace operator $\Delta$ ) and let $B=B(A, R)$ be an open ball contained in $\Omega$. Then, for every $P \in B \backslash B\left(A, \frac{3}{4} R\right)$, we have

$$
\sup \{G(P, Q) ; Q \in \Omega \backslash B(P, R / 2)\} \leq c G(P, A)
$$

where $c$ is a positive constant that depends only on the dimension $d$.

These estimates will be used as follows. Let $f:\left[1, \infty\left[\rightarrow \mathbb{R}_{+}\right.\right.$be a non-negative nonincreasing $C^{2}$ function such that $f(1) \leq 1 / 10,\left|f^{\prime}(t)\right| \leq 1$ and $\left|f^{\prime \prime}(x)\right| \leq c_{0} / t$ for $t>0$ and some positive constant $c_{0}$. Let $\Phi$ denote the closed set of $\mathbb{R}^{d}, \Phi=\left\{\left(t, x^{\prime}\right) ; t \geq 1, x^{\prime} \in \mathbb{R}^{d-1},\left|x^{\prime}\right| \leq f(t)\right\}$. Set $\Omega_{n}=\left\{x \in \mathbb{R}^{d} ; 2^{n}<|x|<2^{n+2}\right\} \backslash \Phi, n \geq 0$. Proposition 4.1 ensures that there exists a positive constant $c$ that depends only on $d$ and $c_{0}$ such that for every integer $n \geq 0$ and every positive $\Delta$-harmonic function $u$ in the region $\Omega_{n}$ that vanishes on $\partial \Omega_{n} \cap \Phi$, one has

$$
u(x) \leq c u\left(A_{n}\right), \quad \forall x \in \Omega_{n} \cap \partial B\left(0,2^{n+1}\right) .
$$

Proof. Set $F=\left\{x \in \Omega_{n} ;|x|=2^{n+1}\left(1 \pm \frac{1}{4}\right)\right\}$. The réduite $\widehat{R}_{u}^{F}$ with respect to $\Omega_{n}$ is the Green potential in $\Omega_{n}$ of a non-negative measure supported by $F$. By this integral representation of $u$ and by homogeneity, it suffices to show that the Green function $G$ of $\Omega_{0}$ satisfies the following inequalities: for each $P \in \Omega_{0} \cap \partial B\left(0,2\left(1 \pm \frac{1}{4}\right)\right)$,

$$
G(Q, P) \leq c G\left(A_{0}, P\right), \quad Q \in \Omega_{0} \cap \partial B(0,2)
$$


with a constant $c$ depending only on $d$ and $c_{0}$. For $P$ sufficiently near to $\Phi$, this follows from Proposition 4.1 and the geometrical assumptions on $\Phi$ (using balls $B \subset \Omega_{0}$ tangent to $\Phi$ ). For other $P$ in $\Omega_{0} \cap \partial B\left(0,2\left(1 \pm \frac{1}{4}\right)\right.$ ) the desired result follows from Harnack inequalities.

\section{2.}

We also require the following lemma (with its corollary) about zero order perturbations of elliptic operators. Let $\Omega$ be a domain in $\mathbb{R}^{d}$, let $U$ be an open relatively compact subset of $\Omega$ that is unthin at each point of $\partial U$ and such that $\Omega^{\prime}=\Omega \backslash \bar{U}$ is connected and contains $O$. Let $L \in \Lambda(\Omega)$ (see beginning of Section 3).

LEMMA 4.2. Let $\left\{\varphi_{n}\right\}_{n \geq 1}$ be an increasing sequence of non-negative functions in $C_{0}^{\infty}(U)$ such that $\lim _{n \rightarrow \infty} \varphi_{n}(x)=+\infty$ in $U$. For each $n \geq$ 1 , let $f_{n}$ be a given positive $\left(L-\varphi_{n} 1\right)$-harmonic function in $\Omega$ satisfying $f_{n}(O)=1$. Then, $f_{n} \rightarrow 0$ uniformly in $\bar{U}$, and for every subsequence $\left\{f_{k_{n}}\right\}$ converging pointwise in $\Omega^{\prime}$, the limit $f=\lim _{n \rightarrow \infty} f_{k_{n}}$ is a non-negative $L$ harmonic function in $\Omega^{\prime}$ vanishing on $\partial \Omega^{\prime} \cap \bar{U} \subset \partial U$. Moreover $f_{k_{n}} \rightarrow f$ uniformly on each bounded subset $A$ of $\Omega^{\prime}$ such that $\bar{A} \subset \Omega$.

Proof. Fix an open relatively compact subset $U^{\prime}$ of $\Omega$ which contains $\bar{U}$. By the Harnack inequalities $f_{n}$ is bounded by a constant $C$ independent of $n$ on $\partial U^{\prime}$ (and hence also in $U^{\prime}$ ). Let $g_{n}$ denote the solution to the Dirichlet problem $L\left(g_{n}\right)-\varphi_{n} g_{n}=0$ in $U^{\prime}$ and $g_{n}=C$ on $\partial U^{\prime}$. The functions $g_{n}$ are smooth in $U^{\prime}$ and by the maximum principle, the $g_{n}$ are decreasing and $0 \leq f_{n} \leq g_{n}$. Clearly, $w=\inf \left\{g_{n} ; n \geq 1\right\}$ is $\left(L-\varphi_{n}\right.$.)-subharmonic (and u.s.c.) in $U^{\prime}$ for every $n \geq 1$; thus $\varphi_{n} . w$ is bounded in $L^{1}(U)$ and $w=0$ a.e. in $U$. The function $w$ being finely continuous (ref. [Her], Bre]) in $U^{\prime}$ (the $L$-fine topology and the $\Delta$-fine topology coincide [Her]) it follows that $w=0$ in $\bar{U}$. This implies that $f_{n} \rightarrow 0$ uniformly in $\bar{U}$ and from that point the other assertions of the lemma are clear.

Corollary 4.3. Let $\Omega, L, U$ and $\Omega^{\prime}$ be as above. Let $A \subset \Omega^{\prime}$ be such that $\bar{A} \subset \subset \Omega$ and let $\varphi \in C_{0}^{\infty}\left(\mathbb{R}^{d}\right)$ be $\geq 0$ and such that $\{\varphi>0\}=U$. Assume that for each positive L-harmonic function $u$ in $\Omega^{\prime}$ vanishing on $\partial U$ we have $u \leq c_{0} u(O)$ in $A$ for some constant $c_{0}>0$. Then for $\lambda$ sufficiently large one has, for each positive $L-\lambda \varphi$-harmonic function in $\Omega$,

$$
u(x) \leq\left(1+c_{0}\right) u(O)
$$

when $x \in A \cup \bar{U}$. 


\section{3.}

Finally we record two simple facts about perturbations of the first order.

LEMMA 4.4. Let $\omega=\left\{\left(x_{1}, x^{\prime}\right) \in \mathbb{R}^{d} ; a<x_{1}<b,\left|x^{\prime}\right|<f\left(x_{1}\right)\right\}$ where $a<b$ and $f:[a, b] \rightarrow \mathbb{R}_{+}$is $>0$ and continuous. Let $a^{\prime}$, $b^{\prime}$ be reals such that $a \leq a^{\prime}<b^{\prime} \leq b$. For $\varphi \in C\left(\mathbb{R}^{d}\right)$, denote $u_{\varphi}$ the harmonic measure in $\omega$ of $\partial \omega \cap\left\{x_{1}=b\right\}$ with respect to $\Delta-\varphi \frac{\partial}{\partial x_{1}}$ and let $K=\left\{x \in \omega ; x_{1}=\right.$ $\left.\left(a^{\prime}+b^{\prime}\right) / 2\right\}$. Then for each $\varepsilon>0$, there is a $C \geq 0$ such that whenever $\varphi \geq 0$ in $\omega$ and $\varphi \geq C$ in $\left\{x \in \omega ; a^{\prime}<x_{1}<b^{\prime}\right\}$, we have $u_{\varphi} \leq \varepsilon$ in $x \in K$.

Proof. To prove this lemma, we may assume that $b^{\prime}=b$ (by maximum principle). Consider the function $v$ in $\mathbb{R}^{d}$ such that $v(x)=x_{1}-a$ for $x_{1} \leq a^{\prime}$ and $v(x)=C^{-1}\left[e^{C\left(x_{1}-a^{\prime}\right)}-1\right]+a^{\prime}-a$ for $x_{1} \geq a^{\prime}$. This function $v$ is positive and superharmonic in $\omega$ with respect to $\Delta-\varphi \frac{\partial}{\partial x_{1}}$ if $\varphi$ and $C$ are as in the statement (with $b^{\prime}=b$ ) and, for $x_{1}=b, v(x) \geq m_{a, b}(C):=$ $C^{-1}\left[e^{C\left(b-a^{\prime}\right)}-1\right]$. Hence, $u_{\varphi}(x) \leq m_{a, b}(C)^{-1} v(x)$ in $\omega$ and the lemma follows.

The next lemma is similar in character.

Lemma 4.5. Let $\omega=\left\{x \in \mathbb{R}^{d} ; x_{1}>1,\left|x^{\prime}\right|<f\left(x_{1}\right)\right\}$ where $f$ : $\left[1,+\infty\left[\rightarrow \mathbb{R}_{+}\right.\right.$is $>0$ and continuous and let $F=\partial \omega \cap\left\{x_{1}=1,\left|x^{\prime}\right| \leq\right.$ $\left.\frac{1}{2} f(1)\right\}$. Consider operators of the form $\mathcal{L}=\Delta-V \partial_{x_{1}}$ with $V$ non-negative in $\omega$. For each $\alpha \in(0,1)$, there exists a smooth non-negative function $V_{0}$ in $\mathbb{R}^{d}$ vanishing outside $\omega$ and such that, whenever $V \geq V_{0}$ in $\omega$, the $\mathcal{L}$ harmonic measure of $F$ in $\omega$ at $(t, 0, \ldots, 0) \in \mathbb{R}^{d}$ is larger than $\alpha$ for all $t \geq 1$.

Proof. By maximum principle, we may assume that $f$ is strictly decreasing. It suffices then to see that in the region

$$
U_{n}=\left\{x \in \mathbb{R}^{d} ; n<x_{1}<n+2,\left|x^{\prime}\right|<\frac{1}{2} f(n+2)\right\}
$$

the $\Delta-C_{n} \partial_{x_{1}}$-harmonic measure of $F_{n}=\left\{\left(x_{1}, x^{\prime}\right) ; x_{1}=n,\left|x^{\prime}\right|<\frac{1}{2} f(n+\right.$ $2)\}$ is larger than $1-\varepsilon_{n}$ on $F_{n+1} \cup\left\{t e_{1} ; n<t \leq n+1\right\}$, where $\varepsilon_{n}=$ $(1-\alpha) / 2^{n}$, for $C_{n}$ large enough. This will follow from a standard comparison argument (but is also clear by a probabilistic argument using Girsanov formula). Fix a smooth positive function $\theta_{n}$ in the ball $\bar{B}\left(0, \frac{1}{2} f(n+2)\right)$ of $\mathbb{R}^{d-1}, \theta_{n}$ being harmonic outside some ball $B\left(0, r_{n}\right)$ with $r_{n}<\frac{1}{2} f(n+2)$ and such that: $0<\theta_{n} \leq 1, \theta_{n}=1$ in a neighborhood of $\bar{B}\left(0, \frac{1}{2} f(n+3)\right)$, 
and $\theta_{n}=0$ on $\partial B\left(0, \frac{1}{2} f(n+2)\right)$. Let $\lambda_{n}>0$ be such that $\Delta \theta_{n} \geq-\lambda_{n} \theta_{n}$ and consider

$$
u_{n}(x)=\left(e^{2 \beta}-e^{\beta\left(x_{1}-n\right)}\right) \theta_{n}\left(x^{\prime}\right), \quad x \in U_{n} .
$$

The function $u_{n}$ is $\Delta-C_{n} \partial_{x_{1}}$.-subharmonic in $U_{n}$ if $-\left(\lambda_{n}+\beta^{2}\right)\left(e^{2 \beta}-1\right)+$ $C_{n} \beta \geq 0$. The largest solution $\beta=\beta\left(\lambda_{n}, C_{n}\right)$ tends to $+\infty$ as $C_{n} \rightarrow \infty$, and for this value of $\beta$ the $\Delta-C_{n} \partial_{x_{1}}$.-harmonic measure of $F_{n}$ in $U_{n}$ is larger than $u_{n} /\left(e^{2 \beta}-1\right)$. The result follows.

\section{§5. Proof of Theorem 2}

\section{1 .}

Let $\Phi=\left\{\left(t, x^{\prime}\right) ; t \geq 1, x^{\prime} \in \mathbb{R}^{d-1},\left|x^{\prime}\right| \leq \alpha e^{-t}\right\}$ with say $0<\alpha \leq 1 / 10$. From Wiener's criteria and a Kelvin transform, it follows that $\Phi$ is thin at infinity w.r. to $\Delta$. Let $U_{0}=\left\{\left(t, x^{\prime}\right) ; t \geq 1, x^{\prime} \in \mathbb{R}^{d-1},\left|x^{\prime}\right|<\alpha e^{-t} / 2\right\}$.

By Lemma 4.5 above, we can construct and fix a smooth non-negative function $V_{0}$ vanishing outside $U_{0}$ and such that for each elliptic operator $\Delta-V \partial_{x_{1}}$ with $V$ smooth and $V \geq V_{0}$, the harmonic measure of $\partial U_{0} \cap$ $B\left(e_{1}, \alpha / 4 e\right)$ in $U_{0}$ is larger than $1 / 2$ on the axis of $U_{0}$.

\section{2.}

Define for each integer $n \geq 1$ :

$$
\begin{gathered}
T_{n}=\left\{\left(t, x^{\prime}\right) \in \Phi ;\left(1-\frac{1}{9}\right) 2^{n} \leq t \leq\left(1+\frac{1}{9}\right) 2^{n}\right\}, \\
T_{n}^{i}=\left\{\left(t, x^{\prime}\right) \in U_{0} ;\left(1-\frac{1}{16}\right) 2^{n}<t<\left(1+\frac{1}{16}\right) 2^{n}\right\}, \\
T_{n}^{e}=\left\{\left(t, x^{\prime}\right) \in \Phi ; \frac{\alpha}{2} e^{-t} \leq\left|x^{\prime}\right| \leq \alpha e^{-t},\left(1-\frac{1}{8}\right) 2^{n} \leq t \leq\left(1+\frac{1}{8}\right) 2^{n}\right\} .
\end{gathered}
$$

We will construct an elliptic operator in the form $L=\Delta-V \frac{\partial}{\partial x_{1}}-\gamma$. where $V$ and $\gamma$ are smooth non-negative functions in $\mathbb{R}^{d}$ such that $V \geq V_{0}$, $V=V_{0}$ outside the "cells" $T_{n}$ and $\gamma=0$ outside the "skins" $T_{n}^{e}$. Moreover $\mathcal{L}$ should satisfy the assumptions of Proposition 3.1.

\section{3.}

We first choose $V$ in the cell $T_{n}, n \geq 1$, such that for every positive $\left(\Delta-V \frac{\partial}{\partial x_{1}} \cdot-\gamma \cdot\right)$-harmonic function $u$ in $\omega_{n}=\left\{x \in \mathbb{R}^{d} ;|x|<(1-\right.$ $\left.\left.\frac{1}{8}\right) 2^{n+1}, x \notin T_{n}^{e}\right\}$ vanishing on $\partial T_{n}^{e}$,-and independently from the choices of $V(\operatorname{resp} . \gamma)$ in the $T_{k}\left(\operatorname{resp} . T_{k}^{e}\right) k<n-$, we have

$$
u(x) \leq C u\left(A_{n}\right), \quad x \in \partial B\left(O, 2^{n}\right) \cap \omega_{n}
$$


for some constant $C \geq 1$ independent from $n$ and $u$. (Recall that $V_{0}$ is fixed, that $V \geq V_{0}$ and that $V=V_{0}$ outside $T_{m}, m \geq 1$.)

Let $u$ be such a function. By Section 4 above - see (4.1)-, there is a constant $C_{1}=C(d)$ such that

$$
u(x) \leq C_{1} u\left(A_{n}\right)
$$

when $x \in \partial B\left(0,2^{n}\right) \backslash \Phi$. On the other hand, if $x \in \partial B\left(0,2^{n}\right) \cap T_{n}^{i}$, it follows from the maximum principle applied in the region $\omega_{n}^{\prime}=\left(B\left(0,2^{n}\right) \backslash T_{n}^{e}\right) \cup$ $\left(U_{0} \cap\left\{x_{1}<\left(1+\frac{1}{8}\right) 2^{n}\right\}\right.$ that

$$
u(x) \leq C_{1} u\left(A_{n}\right)+\psi(x) \max \left\{u(z) ; z \in F_{n}\right\}
$$

where $F_{n}=\left\{z=\left(z_{1}, z^{\prime}\right) \in U_{0} ; z_{1}=2^{n}\left(1+\frac{1}{8}\right)\right\}$ and $\psi(x)$ is the harmonic measure of $F_{n}$ with respect to $L=\Delta-V \frac{\partial}{\partial x_{1}} .-\gamma$. and the domain $\omega_{n}^{\prime}$.

Let $A_{n}^{\prime}=\left(1+\frac{1}{8}\right) 2^{n}$. By standard boundary Harnack inequalities [An1], we have that $u(x) \leq c_{n}^{\prime} u\left(A_{n}^{\prime}\right)$ in $F_{n}$ for a constant $c_{n}^{\prime}$ depending on $n$ (in fact $c_{n}^{\prime}$ depends on the choice of $V_{0}$ in $B\left(A_{n}^{\prime}, 1\right)$ but not on the choice of $V$ in the cells $T_{k}, k \geq 1$ ). On combining this with standard local Harnack inequalities for $\Delta-V_{0} \frac{\partial}{\partial x_{1}}$, we get

$$
u(x) \leq\left[C_{1}+c_{n} \psi(x)\right] u\left(A_{n}\right)
$$

where $c_{n}$ depends solely on $n$ and the dimension $d$.

Now by Lemma 5.1 below, if we choose (and fix) $V=V_{0}+W_{n}$ in $T_{n}$ with $W_{n} \in C_{0}^{\infty}\left(T_{n}\right)$ non-negative and sufficiently large in $T_{n}^{i}$ we shall have $\psi(x) \leq C_{1} / c_{n}$. Thus $u(x) \leq 2 C_{1} u\left(A_{n}\right)$ and (5.1) follows.

LEMMA 5.1. Let $\psi_{W}$ denotes the harmonic measure of $F_{n}$ in $\omega_{n}^{\prime}$ with respect to the operator $L_{W}=\Delta-\left(V_{0}+W\right) \frac{\partial}{\partial x_{1}}$, where $W$ is a smooth non-negative function supported by $T_{n}$. Then, $m_{W}(x)=\sup \left\{\psi_{W}(x) ; x \in\right.$ $\left.T_{n}^{i}, x_{1}=2^{n}\right\} \rightarrow 0$ as $\min \left\{W(x) ; x \in T_{n}^{i}\right\} \rightarrow \infty$.

Proof. Let $f$ be the $\Delta-V_{0} \frac{\partial}{\partial x_{1}}$ harmonic function in $\widetilde{U}=\{x=$ $\left.\left(x_{1}, x^{\prime}\right) \in U_{0} ;\left(1-\frac{1}{8}\right) 2^{n}<x_{1}<\left(1-\frac{1}{9}\right) 2^{n}\right\}$ defined by the boundary values $f(x)=0$ for $x \in \partial \widetilde{U} \cap \partial U_{0}$ and $f(x)=1$ in $\partial \widetilde{U} \cap U_{0}$. Standard barrier arguments (and Harnack inequalities) shows that

$$
\sup \left\{f(x) ; x \in \widetilde{U}, x_{1}=\left(1-\frac{1}{8}+\frac{1}{144}\right) 2^{n}\right\} \leq 1-\delta_{n}
$$


where $\delta_{n}>0$ depends on the choice of $V_{0}$. In particular, using again maximum principle,

$$
\sup \left\{\psi_{W}(x) ; x \in U_{0}, x_{1}=\left(1-\frac{1}{8}\right) 2^{n}\right\} \leq\left(1-\delta_{n}\right) m_{W} .
$$

Let $\pi_{W}$ denotes the $L_{W}$-harmonic measure of $\left\{x_{1}=2^{n}\left(1+\frac{1}{8}\right)\right\}$ in the region $\widetilde{T}_{n}=\left\{x \in U_{0} ;\left(1-\frac{1}{8}\right) 2^{n}<x_{1}<\left(1+\frac{1}{8}\right) 2^{n}\right\}$. Clearly, $\pi_{W}=\psi_{W}-H_{\psi_{W}}$ where $H_{\psi_{W}}$ is $L_{W}$-harmonic in $\widetilde{T}_{n}, H_{\psi_{W}}=\pi_{W}$ in $\partial \widetilde{T}_{n} \cap\left\{x_{1}=\left(1-\frac{1}{8}\right) 2^{n}\right\}$ and vanishes elsewhere in $\partial \widetilde{T}_{n}$. Thus (using again the maximum principle) we have $m_{W} \leq \sup _{F_{n}^{\prime}} \pi_{W}+\left(1-\delta_{n}\right) m_{W}$ where $F_{n}^{\prime}=\left\{x \in U_{0} ; x_{1}=2^{n}\right\}$, or

$$
m_{W} \leq \frac{1}{\delta_{n}} \sup \left\{\pi_{W}(x) ; x \in F_{n}^{\prime}\right\} .
$$

The lemma follows now from Lemma 4.3.

\section{4 .}

Finally we choose the killing term $\gamma$ in $T_{n}^{e}$ : we just need to observe that by Corollary 4.3 and the above construction, if $\gamma$ is chosen sufficiently large in $T_{n}^{e}$ we shall have $u(x) \leq 3 C u\left(A_{n}\right)$ for $x \in \partial B\left(0,2^{n}\right)$ for all $u$ positive and $L$-harmonic in $B\left(0,2^{n+1}\right)$ (regardless to the choice of $\gamma$ in $T_{k}^{e}$ for $k \neq n$ ).

\section{5 .}

We have now constructed an elliptic operator $L=\Delta-V \frac{\partial}{\partial x_{1}} \cdot-\gamma \cdot$, with $V$ and $\gamma$ non-negative and smooth, vanishing outside $\Phi$; moreover, we have in $U_{0}, \gamma=0, V \geq V_{0}$ and $L$ satisfies the assumptions of Proposition 3.1. Whence:

a) By Proposition 3.1, $\left(\mathbb{R}^{d}, L\right)$ is a transient pair (thus, there exists a Green's function $G_{L}$ for $L$ over $\mathbb{R}^{d}$ ) whose Martin boundary is reduced to a point, i.e. there exists a positive $L$-harmonic function $h$ on $\mathbb{R}^{d}$ and $h$ is unique up to a multiplication by a positive constant. Moreover $h$ is bounded in $\mathbb{R}^{d}$.

b) By a standard maximum principle, $G_{L}(x, O) \geq c v(x)$ where $v(x)$ is the $L$-harmonic measure, inside $U_{0}$ and at $x$, of $B\left(e_{1}, \alpha / 4 e\right) \cap \partial U_{0}$. Hence, by the choice of $V_{0}$ and $V$, it follows that $\inf \left\{G_{L}\left(t e_{1}, 0\right) ; t>0\right\}>0$.

\subsection{End of proof of Theorem 2}

Using a standard relativisation procedure, we introduce now the operator $\mathcal{L} \in \Lambda\left(\mathbb{R}^{d}\right)$ such that $\mathcal{L}(u)=h^{-1} L(u h)$ for $u \in C^{2}\left(\mathbb{R}^{d}\right)$. Clearly this operator is in the form $\mathcal{L}=\Delta .+D . \nabla$ where $D$ is a smooth vector field 
in $\mathbb{R}^{d}$. Moreover, it follows from what has been established for $L$ that: (i) the pair $(M, \mathcal{L})$ is Greenien (and $G_{\mathcal{L}}(x, y)=\frac{h(y)}{h(x)} G_{L}(x, y)$ ), (ii) the functions $x \mapsto G_{\mathcal{L}}(x, y)$ do not vanish at infinity, but (iii) positive $\mathcal{L}$-harmonic functions in $\mathbb{R}^{d}$ are the positive constant functions.

\section{7.}

Theorem 2 and its proof can be extended to the case $d=2$. To show this, one may adapt the above construction on starting with an elliptic operator $L_{0}=\Delta .+D_{0} \cdot \nabla$. (rather than $\left.L_{0}=\Delta\right)$ where $D_{0}=\left(D_{0,1}, D_{0,2}\right)$ is a Lipschitz continuous vector field in $\mathbb{R}^{2}$ such that: $D_{0}(x)=x /|x|^{2}$ if $|x| \geq 1$ and $x_{1}<0, D_{0}(x)=x_{2}^{-1}(0,1)$ if $|x| \geq 1, x_{1} \geq 0$ and $e^{-x_{1}} / 10 \leq\left|x_{2}\right|$. It is also required that $D_{0,1}\left(x_{1}, x_{2}\right)=D_{0,1}\left(x_{1},-x_{2}\right)=0$ and $D_{0,2}\left(x_{1}, x_{2}\right)=$ $-D_{0,2}\left(x_{1},-x_{2}\right) \geq 0$ when $x_{2} \geq 0$ and $x_{1} \geq 1$. It can be shown that $L_{0}$ is transient, that it satisfies (as the standard Laplacian) uniform Harnack inequalities on large balls (thus, $L_{0}$ Martin's boundary is reduced to one point) and that the set $\Phi$ (defined as above) is $L_{0}$ thin at infinity. But we shall not discuss further the construction here.

\section{§6. Critical points of certain harmonic functions with periodicity properties}

In this section we specify the second question -due to G. Benarous and H. Owhadi- which will be considered in this paper. Let $d$ denote an integer $\geq 1$ and let $\varphi$ be a real $C^{\infty}$ function in $\mathbb{R}^{d}$ which is $\mathbb{Z}^{d}$-periodic, i.e. such that $\varphi\left(x+e_{i}\right)=\varphi(x)$ for $x \in \mathbb{R}^{d}$ and every vector $e_{i}, 1 \leq i \leq d$, from the canonical basis of $\mathbb{R}^{d}$. Then, as explained below, for each $i \in\{1, \ldots, d\}$, there exists a unique function $F_{i}$ in $\mathbb{R}^{d}$, satisfying $F_{i}(0)=0, F_{i}\left(x+e_{j}\right)=$ $F_{i}(x)+\delta_{i, j}$ for $x \in \mathbb{R}^{d}, j \in\{1, \ldots, d\}$, and

$$
\Delta F_{i}-\nabla \varphi \cdot \nabla F_{i}=0
$$

The question raised by G. Benarous and H. Owhadi reads then as follows.

$(Q)$ : is the map $F: x \mapsto F(x)=\left(F_{1}(x), \ldots, F_{d}(x)\right)$ a diffeomorphism of $\mathbb{R}^{d}$ onto itself?

Remark 6.1. 1. The functions $G_{i}(x)=x_{i}-F_{i}(x)$ are periodic and thus bounded. So $\lim _{\|x\| \rightarrow \infty}\|F(x)\|=+\infty$. Since $\mathbb{R}^{d}$ is simply connected, this means that $F$ is a diffeomorphism of $\mathbb{R}^{d}$ onto $\mathbb{R}^{d}$ if and only if the Jacobian 
of $F$ is non degenerated at every $x \in \mathbb{R}^{d}$. 2. If $F$ is a diffeomorphism of $\mathbb{R}^{d}$, it is easily seen, using the relation $F(x+z)=F(x)+z$ for $z \in \mathbb{Z}^{d}$, that $\|F(x)-F(y)\| \geq C\|x-y\|$ for some constant $C>0$ and all $x, y$ in $\mathbb{R}^{d}$. Conversely, if for a given dimension $d$ it is true that for every $F$ (constructed as above from some $\varphi$ smooth and $\mathbb{Z}^{d}$-periodic in $\mathbb{R}^{d}$ ) one has $\|F(x)\| \geq C\|x\|, x \in \mathbb{R}^{d}$, for some $C>0$, then every such $F: \mathbb{R}^{d} \rightarrow \mathbb{R}^{d}$ is a local diffeomorphism at every $a \in \mathbb{R}^{d}$ (using $x \mapsto F(x+a)-F(a)$ ). In fact, the original question of $\mathrm{G}$. Benarous and H. Ohwadi was: is it true that $F$ must satisfy $\|F(x)\| \geq C\|x\|, x \in \mathbb{R}^{d}$, for some $C=C_{\varphi}>0$ ?

To justify existence and uniqueness of $F_{i}$, it suffices -setting $F_{i}(x)=$ $x_{i}-G_{i}(x)$ - to show existence and uniqueness of $G_{i}: \mathbb{R}^{d} \rightarrow \mathbb{R}$ smooth, $\mathbb{Z}^{d}$-periodic and such that

$$
\Delta G_{i}-\nabla \varphi \cdot \nabla G_{i}=-\partial_{i} \varphi
$$

in $\mathbb{R}^{d}$. By the strong maximum principle, the periodic solutions $u$ of $\Delta u-\nabla \varphi \cdot \nabla u=0$ are the constant functions, whence the uniqueness of $G_{i}$. On the other hand, if we set $v=u \exp (-\varphi / 2)$ and $\psi=\exp (-\varphi / 2)$, the equation $\Delta u-\nabla \varphi \cdot \nabla u=-\partial_{i} \varphi$ is equivalent to the equation (with smooth and periodic coefficients) $\Delta v-v \Delta \psi / \psi=2 \partial_{i} \psi$. The elliptic operator $v \mapsto \Delta v-v \Delta \psi / \psi$ is self-adjoint on the torus $T^{d}=\mathbb{R}^{d} / \mathbb{Z}^{d}$ and its kernel is reduced to the multiple of $\psi$ (since periodic solutions $u$ of $\Delta u-\nabla \varphi \cdot \nabla u=0$ are constant). Moreover the right hand side $2 \partial_{i} \psi$ is orthogonal in $L^{2}\left(T^{d}\right)$ to $\psi: \int_{T^{d}} \psi(x) \partial_{i} \psi(x) d x=\frac{1}{2} \int_{[0,1]^{d}} \partial_{i}\left(\psi(x)^{2}\right) d x=0$. Thus Fredholm's theory provides the existence of $G_{i}$.

\section{§7. The case $d=2$}

In this section, it is shown that for $d \leq 2$, question $(Q)$ above always admits a positive answer. The case $d=1$ is elementary.

TheOREM 3. If $d=2$, the map $F: \mathbb{R}^{2} \rightarrow \mathbb{R}^{2}$ defined in Section 6 is a diffeomorphism of $\mathbb{R}^{2}$ onto $\mathbb{R}^{2}$.

It suffices to show that for each $u \in \mathbb{R}^{2},\|u\|=1$, the function $F_{u}(m)=$ $F_{1}(m) u_{1}+F_{2}(m) u_{2}, m \in \mathbb{R}^{2}$, has no critical point. This function is $\Delta-$ $\nabla \varphi . \nabla$ harmonic and satisfies $F_{u}\left(m+k e_{1}+\ell e_{2}\right)=F_{u}(m)+k u_{1}+\ell u_{2}$ for $(k, \ell) \in \mathbb{Z}^{2}$. We shall see that such a function has no critical points.

To begin with, observe that in a strip $T_{R}=\left\{m \in \mathbb{R}^{2} ;|m . u| \leq R\right\}$, $F_{u}$ is bounded: each point $m$ in $T_{R}$ is within a distance $\leq \sqrt{2} / 2$ to a point 
$m^{\prime}=(p, q) \in \mathbb{Z}^{2}$ and $\left|m^{\prime} . u\right| \leq R+\sqrt{2} / 2$; so $F_{u}(m)=F_{u}\left(m-m^{\prime}\right)+m^{\prime} . u$ is bounded.

This also shows that $m . u-C \leq F_{u}(m) \leq m . u+C, m \in \mathbb{R}^{2}$, for some $C>0$. Thus, $F_{u}$ is positive with linear growth in the half-plane $\{m ; m . u>C\}$.

For each $t \in \mathbb{R}$, the set $U=\left\{F_{u}<t\right\}$ is connected: otherwise, since by the above $U$ contains a half-plane $\left\{m \in \mathbb{R}^{2} ; m . u<s\right\}$ and is contained in another half-plane $\left\{m \in \mathbb{R}^{2} ; m . u<s^{\prime}\right\}$, there exists a component $U^{\prime}$ of $U$ contained in a strip $T_{R}$ and $F_{u}=t$ on $\partial U^{\prime}$. Lemma 7.1 below implies that $F_{u}$ is then constant in $U^{\prime}$ and hence constant in $\mathbb{R}^{2}$ by unique continuation for smooth elliptic equations ([Aro], [Hör, p. 14]), a contradiction. Thus, by Lemma 7.2 , we have $\nabla F_{u} \neq 0$ in $\partial U$.

Finally, $t$ being arbitrary, we must have $\nabla F_{u}(m) \neq 0$ at every $m \in \mathbb{R}^{2}$.

LEMMA 7.1. Let $f$ be a real bounded continuous function in a strip $\{x ;-A \leq x . u \leq A\}$, which is nonpositive for $x . u= \pm A$ and $\Delta-\nabla \varphi . \nabla-$ subharmonic in $W_{A}=\{x ;-A<x . u<A\}$. Then, $f(m) \leq 0$ in $W_{A}$.

Proof. Denote $u^{\perp}$ the unit vector in $\mathbb{R}^{2}$ which is directly orthogonal to $u$. Let $m \in W_{A}$ and let $\omega(m, R)$ be the harmonic measure at $m$, of $W_{A} \cap\left\{x ;\left|x-m . u^{\perp}\right|=R\right\}$, in the region

$$
W_{A, R}=\left\{x \in \mathbb{R}^{2} ;-A<x . u<A, m . u^{\perp}-R<x . u^{\perp}<m . u^{\perp}+R\right\}
$$

and with respect to $\Delta-\nabla \varphi \cdot \nabla$. A standard argument involving maximum principle and an iteration procedure shows that $\omega(m, R) \leq C \exp (-\alpha R)$ for $R \geq 1$ and constants $C>0$ and $\alpha>0$. Since $f(m) \leq\|f\|_{\infty} \omega(m, R)$, we have $f(m) \leq 0$ by letting $R \rightarrow \infty$.

LEMma 7.2. Let $f: \mathbb{R}^{2} \rightarrow \mathbb{R}$ be a $\Delta-\nabla \varphi \cdot \nabla$.-harmonic function and let $t$ be a real. If the set $W=\left\{m \in \mathbb{R}^{2} ; f(m)<t\right\}$ is connected, then $\nabla f \neq 0$ in $\partial W$.

Proof. Assume as we may $t=0, f$ non constant, and, arguing by contradiction, that $\nabla f\left(m_{0}\right)=0$ for some point $m_{0} \in \partial W$. The argument is then broken into two steps.

a) Consider the Taylor series of $f()=.f()-.f\left(m_{0}\right)$ at $m_{0}$. As wellknown this series is not identically zero ([Aro], [Hör $]$ ); thus, $f(m)=Q(m-$ $\left.m_{0}\right)+\left\|m-m_{0}\right\|^{p} \varepsilon(m)$ where $Q$ is a non zero homogeneous polynomial of 
degree $p \geq 2$ and where $\varepsilon$ is such that $\lim _{m \rightarrow m_{0}} \varepsilon(m)=0$. It is easily seen that $Q$ is $\Delta$-harmonic: for, if $\lambda>0$, the function $u: \xi \mapsto \lambda^{-p} f\left(m_{0}+\lambda \xi\right)$ solves $\Delta u(\xi)-\lambda \nabla \varphi\left(m_{0}+\lambda \xi\right) . \nabla u(\xi)=0$ and for $\lambda \rightarrow 0$ this function tends to $Q$, uniformly on compacts. This provides the result (using also Schauder's interior estimates).

b) Thus, $Q(z)=\operatorname{Re}\left(c z^{p}\right)$, with $c \in \mathbb{C}^{*}, p \geq 2$. It follows that we may construct four small segments $m_{0} m_{1}, m_{0} m_{2}, m_{0} m_{3}$ and $m_{0} m_{4}$, in the clockwise order around $m_{0}$ and such that: if $F_{1}=\left[m_{0}, m_{1}\right] \cup\left[m_{0}, m_{3}\right]$ and $F_{2}=\left[m_{0}, m_{2}\right] \cup\left[m_{0}, m_{4}\right]$, the function $f$ is $>0$ in $F_{1} \backslash\left\{m_{0}\right\}$ and $<0$ in $F_{2} \backslash\left\{m_{0}\right\}$. Since $W$ is connected, it follows that there exists a simple (polygonal) closed curve through $m_{0}$ in $\{f \leq 0\}$ separating $\{f>0\}$. This implies that $\{f>0\}$ has at least one bounded component $U$. But $f=0$ in $\partial U$, and, by the maximum principle, $f=0$ in $U$ which is absurd.

\section{$\S 8$. The case $d \geq 3$}

In this section we prove the following statement.

THEOREM 4. For each $d \geq 3$, there exists a non-negative function $\varphi \in \mathcal{C}^{\infty}\left(\mathbb{R}^{d}\right)$ which is $\mathbb{Z}^{d}$-periodic and such that the associated function $F_{1}$ in $\mathbb{R}^{d}$ (see Section 6 ) has critical points.

The case $d \geq 4$ follows from the case $d=3$ by adding dummy variables. So we restrict to $d=3$.

\subsection{The regions $V_{0}$ and $V_{1}$}

Let $C$ denote the closed half-circle in $\mathbb{R}^{3}$ consisting of all points $(x, y, z)$ $\in \mathbb{R}^{3}$ such that $z=0, x \geq 0$, and $x^{2}+y^{2}=(1 / 3)^{2}$. Denote $\Gamma$ the group of translations of $\mathbb{R}^{3}$ of the form $\xi \mapsto \xi+m e_{2}+n e_{3},(m, n) \in \mathbb{Z}^{2}$. For $\gamma \in \Gamma$, set $C_{\gamma}=\gamma(C)$.

Let $F$ denote the union of the plane $x=0$ and of all the half-circles $C_{\gamma}$, $\gamma \in \Gamma$. For $t \in] 0,1 / 10\left[\right.$, set $U_{t}=\left\{m=(x, y, z) \in \mathbb{R}^{3} ; x>0, d(m, F)<t\right\}$. Clearly, $U_{t}$ is the union of the slice $\{0<x<t\}$ and of the open half-torus $T_{\gamma}, \gamma \in \Gamma$, of soul $C_{\gamma}$ and radius $t$ around $C_{\gamma}$.

Set $f(m)=d(m, F)$ and let $\tilde{f}=f * \theta$, be a regularization of $f$, where $\theta \in C_{0}^{\infty}\left(\mathbb{R}^{3}\right)$ is a decreasing function of the distance to the origin supported by a ball of radius $\varepsilon^{\prime}<1 / 100$. Set $\widetilde{U}_{t}=\{m=(x, y, z) ; x>0, \tilde{f}(m)<t\}$ for $t>0$. Clearly, $\widetilde{U}_{1 / 16} \subset U_{1 / 8}$. By Sard's theorem we may choose $\varepsilon \in] 1 / 32,1 / 16\left[\right.$, such that $\widetilde{U}_{\varepsilon}=\{0<\tilde{f}<\varepsilon\} \cap\{x>0\}$ have a smooth 
boundary (it consists of the plane $x=0$ and of a $\Gamma$-invariant surface which is close to $\left.\partial U_{\varepsilon} \cap\{x>0\}\right)$.

Let $V_{0}=\widetilde{U}_{\varepsilon}$ and let $V_{1}$ denote the image of $V_{0}$ under the isometry $\Phi:(x, y, z) \mapsto\left(\frac{1}{2}-x, z, y\right)$. Note that $V_{1}$ is disjoint from $V_{0}$, that $V_{0}$ contains the half-circles $C_{\gamma}$ deprived from their ends and that $V_{1}$ contains the open half-circles $C_{\gamma}^{\prime}=\gamma(\Phi(C))$ with ends removed. Thus, the construction is such that each half-circle $C_{\gamma}$ is not homotopic in $\left([0,1 / 2] \times \mathbb{R}^{2}\right) \backslash C_{\gamma}^{\prime}$, by an homotopy with fixed ends, to a path contained in the plane $x=0$.

\section{2.}

We now pause to state an approximation lemma which will be needed to construct the function $\varphi$ in our example. Let $\bar{M}$ be a smooth compact Riemannian manifold with a boundary $\partial M$ written as the union of two disjoint nonempty compact subsets $\Sigma_{0}$ and $\Sigma_{1}$. Set $M=\bar{M} \backslash \partial \bar{M}$, $\delta(x)=d\left(x, \Sigma_{0}\right)$ for $x \in \bar{M}$ and fix a sequence $\left\{\varphi_{j}\right\}$ of non-negative functions in $\bar{M}$ of the form $\varphi_{j}(x)=\theta_{j}(\delta(x))$ where $\theta_{j}$ is a $C^{2}$ non-negative and nonincreasing function in $\left[0, \infty\left[\operatorname{such}\right.\right.$ that: $\operatorname{supp}\left(\theta_{j}\right) \subset\left[0, \varepsilon_{j}\right], \lim _{j \rightarrow \infty} \varepsilon_{j}=0$, $\lim _{j \rightarrow \infty} \int_{0}^{\varepsilon_{j}} \exp \left(\theta_{j}(\tau)\right) d \tau=+\infty$.

Then, the following holds.

Proposition 8.1. Let $f \in C\left(\Sigma_{1} ; \mathbb{R}\right)$ and let $\left\{u_{j}\right\}$ be a sequence of continuous functions in $\bar{M}$ which are $C^{2}$ in $M$ and such that (i) $\Delta u_{j}-$ $\nabla \varphi_{j} . \nabla u_{j}=0$ in $M$ (ii) $u_{j}=f$ in $\Sigma_{1}$, and (iii) $\left\{u_{j}\right\}$ is uniformly bounded in $M$. Then, as $j \rightarrow+\infty, u_{j}$ converges to the function $\tilde{u}$ which solves $\Delta \tilde{u}=0$ in $M, \tilde{u}=f$ in $\Sigma_{1}, \partial_{n} u=0$ in $\Sigma_{0}$, the convergence being uniform on every compact subset of $M \cup \Sigma_{1}$.

We have denoted $\partial_{n} u$ the inner normal derivative of $u$ along $\Sigma_{0}$. The proof of Proposition 8.1 is given in Section 8.5. Note that for our construction, we only require the first step of this proof i.e. the case $f=0$.

\section{3.}

We also need the following elementary lemma whose proof is omitted.

LEMmA 8.2. Suppose that $\varphi \in C^{\infty}\left(\mathbb{R}^{3}\right)$ is $\mathbb{Z}^{3}$-periodic and even with respect to $x_{1}$ (i.e. $\varphi\left(x_{1}, x_{2}, x_{3}\right)=\varphi\left(-x_{1}, x_{2}, x_{3}\right)$ for $\left.x \in \mathbb{R}^{3}\right)$. Then the solution $F_{1}$ of $\Delta F_{1}-\nabla \varphi \cdot \nabla F_{1}=0$ which is $\mathbb{Z}^{2}$-periodic in $\left(x_{2}, x_{3}\right)$, zero at 0 and such that $F_{1}\left(x+e_{1}\right)=1+F_{1}(x)$, is odd with respect to $x_{1}$ : $F_{1}\left(x_{1}, x_{2}, x_{3}\right)=-F_{1}\left(-x_{1}, x_{2}, x_{3}\right)$ for $x \in \mathbb{R}^{3}$. 


\subsection{End of the example's construction}

We first choose a function $\varphi: Y=[0,1 / 2] \times \mathbb{R}^{2} \rightarrow \mathbb{R}_{+}, \Gamma$-periodic, smooth, with support in $] 0,1 / 2\left[\times \mathbb{R}^{2}\right.$ and constant in $Y \backslash\left(V_{0} \cup V_{1}\right)$. We also require that in $V_{0}$ (resp. in $\left.V_{1}\right), \varphi(m)=\theta\left(d\left(m, S_{0}\right)\right)$ (resp. $\varphi(m)=$ $\left.\theta\left(d\left(m, S_{1}\right)\right)\right)$ where $S_{0}=\partial V_{0} \cap\left\{(x, y, z) \in \mathbb{R}^{3} ; 0<x<1 / 2\right\}$ and $S_{1}=$ $\partial V_{1} \cap\left\{(x, y, z) \in \mathbb{R}^{3} ; 0<x<1 / 2\right\}$, where $\theta$ is smooth nonincreasing in $[0,1]$, constant in a neighborhood of 0 and supported in $[0,1 / 100]$. Replacing $\theta(t)$ by $\lambda \theta(\lambda t)$ with $\lambda$ large, Proposition 8.1 says that we may also assume that with respect to the domain $V_{0}$ (resp. $V_{1}$ ) the $\Delta .-\nabla \varphi . \nabla$. harmonic measure of the plane $x=0$ (resp. of the plane $x=1 / 2$ ) is $\geq 9 / 10$ on the $C_{\gamma}\left(\right.$ resp. the $\left.C_{\gamma}^{\prime}\right), \gamma \in \Gamma$.

Extend $\varphi$ by "symmetry" to $Y_{1}=[0,1] \times \mathbb{R}^{2}$ by letting $\varphi(x, y, z)=$ $\varphi(1-x, y, z)$ for $x \in[1 / 2,1]$, and then extend $\varphi$ to $\mathbb{R}^{3}$ by periodicity with respect to the first coordinate, i.e. by letting $\varphi(x, y, z)=\varphi(x-n, y, z)$ for $x \in[n, n+1], n \in \mathbb{Z}$. Clearly $\varphi$ is smooth, $\mathbb{Z}^{3}$-periodic in $\mathbb{R}^{3}$ such that $\varphi(1-x, y, z)=\varphi(x, y, z)$, for $(x, y, z) \in \mathbb{R}^{3}$.

In particular, $\varphi$ is even in the first coordinate. By Lemma 8.2, the corresponding function $F_{1}$ vanishes in the plane $x=0$, and hence is equal to one in the plane $x=1$. A symmetry argument shows that $F_{1}=1 / 2$ on the plane $x=1 / 2$, and the choice of $\theta$ (and $\varphi$ ) ensures that $F_{1}$ is less than $1 / 20$ in $C_{\gamma}$ and larger than $1 / 2-1 / 20$ on $C_{\gamma}^{\prime}, \gamma \in \Gamma$.

It follows that $F_{1}$ admits at least one critical value in the interval $[0,1 / 4]$, since otherwise the plane $x=0$ would be a retract of the set $\left\{F_{1} \leq 1 / 4\right\} \cap\{0 \leq x \leq 1\}$. A fortiori, each $C_{\gamma}$ would be homotopic in $\{0 \leq x<1 / 2\} \backslash C_{\gamma}^{\prime}$ to a curve in the plane $x=0$, by an homotopy with fixed endpoints. A contradiction. Theorem 4 is proven.

\subsection{Proof of Proposition 8.1}

The proof consists in two steps.

Step 1. Let $w_{j}$ denote the harmonic measure of $\Sigma_{0}$ in $M$ with respect to $\Delta$. $-\nabla \varphi_{j} . \nabla$., i.e. $w_{j}$ is continuous in $\bar{M}$, smooth in $M$ and satisfies $\Delta w_{j}-\nabla \varphi_{j} . \nabla w_{j}=0$ in $M, w_{j}=0$ in $\Sigma_{1}, w_{j}=1$ in $\Sigma_{0}$. We will show that $w_{j} \rightarrow 0$ uniformly on every compact subset of $M \cup \Sigma_{1}$. By the maximum principle, it suffices to show that $w_{j} \rightarrow 0$ uniformly in every compact subset of a region $M_{\varepsilon}=\left\{m \in M ; 0<d\left(m, \Sigma_{0}\right)<\varepsilon\right\}$ for some small $\varepsilon>0$. We choose and fix $\varepsilon>0$ so small that (i) $\overline{M_{2 \varepsilon}} \cap \Sigma_{1}=\emptyset$ and (ii) the map $\Sigma_{0} \times[0,2 \varepsilon] \rightarrow M$ which associates to $(x, t)$ the point at distance $t$ from $x$ 
on the geodesic segment emanating from $x$ orthogonally to $\Sigma_{0}$ induces a diffeomorphism from $\Sigma_{0} \times(0,2 \varepsilon)$ onto $M_{2 \varepsilon}$.

Consider now the functions $s_{j}(x)=g_{j}(\delta(x)), x \in \bar{M}_{\varepsilon}$ where $g_{j}$ solves $g_{j}^{\prime \prime}(t)+g_{j}^{\prime}(t)\left(-C-\theta_{j}^{\prime}(t)\right)=0$ in $[0, \varepsilon], g_{j}(0)=1, g_{j}(\varepsilon)=0$. A computation (using that $g_{j}^{\prime} \leq 0$ ) shows that if the constant $C$ is chosen so that $|\Delta \delta| \leq C$ in $\bar{M}_{\varepsilon}$ we have $\Delta s_{j}-\nabla \varphi_{j} . \nabla s_{j} \leq 0$.

Thus, $g_{j}$ is $\Delta .-\nabla \varphi_{j} . \nabla$. superharmonic in $\bar{M}_{\varepsilon}$ and since $s_{j}(x)=$ $\left(\int_{\delta(x)}^{\varepsilon} \exp \left(C \tau+\theta_{j}(\tau)\right) d \tau\right) /\left(\int_{0}^{\varepsilon} \exp \left(C \tau+\theta_{j}(\tau)\right) d \tau\right)$, we see that $s_{j} \rightarrow 0$ uniformly in $\bar{M}_{\varepsilon} \backslash M_{s}, \forall s>0$. This already proves the desired property for the manifold $\bar{M}_{\varepsilon}$ instead of $\bar{M}$.

To conclude this step, observe that $s_{j} \geq w_{j}-H_{j}$ if $H_{j} \in \mathcal{C}\left(\bar{M}_{\varepsilon}\right)$ solves $\Delta H_{j}-\nabla \varphi_{j} . \nabla H_{j}=0$ in $M_{\varepsilon}, H_{j}=w_{j}$ in $S_{\varepsilon}=\partial \bar{M}_{\varepsilon} \cap M, H_{j}=0$ in $\Sigma_{0}$. For any given small $t>0$, the harmonic measure of $\Sigma_{1}$ in $M \backslash \bar{M}_{t}$, with respect to $\Delta .-\nabla \varphi_{j} . \nabla$., is larger than a constant $\eta=\eta(\varepsilon)$ in $S_{\varepsilon}$. Thus, $\sup _{S_{t}} H_{j} \leq \sup _{S_{\varepsilon}} H_{j} \leq \sup _{S_{\varepsilon}} w_{j} \leq(1-\eta) \sup _{S_{t}} w_{j}$ (using Markov property or maximum principle for the last inequality). So, $w_{j} \leq \eta^{-1} \sup _{S_{t}} s_{j}$ in $\bar{M} \backslash M_{t}$. The claim follows.

Step 2. By the first step, the conclusion of the proposition is proven if $f=0$ in $\Sigma_{1}$. In general we may decompose $u_{j}$ as $u_{j}=u_{j}^{0}+\tilde{u}_{j}$ where $\tilde{u}_{j} \in \mathcal{C}(\bar{M}, \mathbb{R})$ is smooth in $M$, satisfies $\Delta \tilde{u}_{j}-\nabla \varphi_{j} . \nabla \tilde{u}_{j}=0$ in $M, \tilde{u}_{j}=f$ in $\Sigma_{1}$ and $\partial_{n} \tilde{u}_{j}=0$ in $\Sigma_{0}$ (this implies that $\left\|\tilde{u}_{j}\right\|_{\infty, \bar{M}} \leq\|f\|_{\infty, \bar{M}}$ ). By Step 1 and maximum principle, we are left with showing that $\tilde{u}_{j} \rightarrow \tilde{u}$ uniformly on each compact subset of $\bar{M} \backslash \Sigma_{1}$.

An obvious perturbation argument shows that we may also assume that $\tilde{u}_{j}$ is smooth in $\bar{M}$ (i.e. $f$ is smooth in $\Sigma_{1}$ ). Moreover, since by the Schauder estimates, the sequence $\left\{\tilde{u}_{j}\right\}$ is bounded in $C^{2,1}(K)$ for every compact $K \subset \bar{M} \backslash \Sigma_{0}$ we may assume that $\left\{\tilde{u}_{j}\right\}$ converges to some function $u$ in $C^{2}\left(\bar{M} \backslash \Sigma_{0}\right)$, and we are left with showing that $u=\tilde{u}$.

Now, for every $\theta \in H^{1}(\bar{M})$ with $\theta=0$ on $\Sigma_{1}$

$$
\int_{M} \nabla \tilde{u}_{j} \cdot \nabla \theta d \sigma+\int_{M} \theta \nabla \tilde{u}_{j} . \nabla \varphi_{j} d \sigma=0
$$

and if we let $\psi=\theta e^{\varphi_{j}}$ we find that $\int_{M} e^{-\varphi_{j}} \nabla \tilde{u}_{j} . \nabla \psi d \sigma=0$. Thus $v=\tilde{u}_{j}$ minimizes the Dirichlet norm $\int_{M} e^{-\varphi_{j}}|\nabla v|^{2} d \sigma$ for $v \in V_{f}=\left\{v \in H^{1}(\bar{M})\right.$; $v=f$ in $\left.\Sigma_{1}\right\}$. 
By Fatou's lemma, it follows from the inequalities $\int_{M} e^{-\varphi_{j}}\left|\nabla \tilde{u}_{j}\right|^{2} d \sigma \leq$ $\int_{M} e^{-\varphi_{j}}|\nabla v|^{2} d \sigma, v \in V_{f}$, that $\int_{M}|\nabla u|^{2} d \sigma \leq \int_{M}|\nabla v|^{2} d \sigma$ (we use the fact that $\left.e^{-\varphi_{j}} \leq 1\right)$. Thus $u \in H^{1}(\bar{M})$, satisfies the Neumann boundary condition $\partial_{n} u=0$ and is harmonic in $M$. Hence $u=\tilde{u}$ in $\bar{M}$.

\section{§9. A stability property for Green's functions}

In this section, we state a result -suggested by another question of Y. Pinchover [Pi2] (see 9.2 below) - which in particular gives a sufficient condition for two Schrödinger operators $L_{i}=\Delta .-V_{i} ., i=1,2$, with large potentials $V_{i}$ to have equivalent Green's functions. The result is mainly an application of [An4] (see also [An5]).

\section{1 .}

Let $(M, g)$ be a complete non compact Riemannian manifold of class $C^{1}$, i.e. the manifold $M$ is $C^{2}$, the metric $g$ is $C^{1}$. It is also assumed that $(M, g)$ has bounded geometry in the sense that Assumption 1.1 in [An4] are satisfied: there are positive reals $r_{0} \leq 1, c_{0} \geq 1$, and a family of charts $\chi_{x}: B\left(x, r_{0}\right) \rightarrow \mathbb{R}^{n}, x \in M$, which are $c_{0}$-bilipschitz $(n=\operatorname{dim}(M))$. Let $O$ be some fixed reference point in $M$ and let $d$ denote the distance in $(M, g)$.

We also fix a function $V_{0}$ of class $C^{1}$ in $M$ such that $V_{0} \geq 1$ and $\left|\nabla V_{0}\right| \leq$ $C_{0} V_{0}^{3 / 2}$ in $M$ for some constant $C_{0}>0$ (the reason for this assumption appears below after Lemma 9.3) and consider two elliptic operators $L_{1}$ and $L_{2}$ in $M$ in the form

$$
L_{j}(u)=\operatorname{div}\left(\mathcal{A}_{j}(\nabla u)\right)+B_{j} \cdot \nabla u-V_{j} u, \quad j=1,2
$$

where the $\mathcal{A}_{j}$ are bounded Borel and uniformly accretive sections of $\operatorname{End}(T(M))$, the $B_{j}$ are Borel vector fields in $M$ such that $\left|B_{j}\right| \leq C_{1} V_{0}^{1 / 2}$ and the $V_{j}$ are Borel functions in $M$ such that $\left|V_{j}\right| \leq C_{1} V_{0}$ in $M$ for $j=1,2$ and for some constant $C_{1}>0$.

Finally we denote $E=V_{0}\left\|\mathcal{A}_{1}-\mathcal{A}_{2}\right\|+\sqrt{V_{0}}\left\|B_{1}-B_{2}\right\|+\left|V_{1}-V_{2}\right|$ and set for $t \geq 0$

$$
\Phi(t)=\sup \left\{\frac{E(x)}{V_{0}(x)} ; d(O, x) \geq t\right\} .
$$

THEOREM 5. Assume that $V_{j} \geq C_{1}^{-1} V_{0}$ outside a compact subset of $M$ and that $L_{j}$ admits a Green function $G_{j}$ in $M$ for $j=1,2$. If moreover

$$
\int_{0}^{\infty} \Phi(R) \max \left\{\sqrt{V_{0}(x)} ; d(O, x)=R\right\} d R<\infty
$$


there exists a constant $C$ such that for all $x$ and $y$ in $M$,

$$
C^{-1} G_{1}(x, y) \leq G_{2}(x, y) \leq C G_{1}(x, y)
$$

Note that if $V_{j} \geq 0$ for $j=1,2$, the existence of $G_{j}$ follows from the other assumptions. The next statement is a corollary of the proof of Theorem 5. The definition of a small perturbation [Pi1] is recalled later (see 9.5 below).

Corollary 9.1. Assume that $V_{1} \geq C_{1}^{-1} V_{0}$ in $M$. If

$$
\int_{0}^{\infty} \frac{\max \left\{\sqrt{V_{0}(x)} ; d(0, x)=R\right\}}{\min \left\{V_{0}(x) ; d(0, x) \geq R\right\}} d R<\infty .
$$

Then -1 is a small perturbation of $L_{1}$.

\section{2 .}

In particular, let $H=\Delta-V$ be a Schrödinger operator on $R^{d}$ such that $V(x)=Q(|x|)$ for $x \in \mathbb{R}^{d}$, with $Q:[0, \infty) \rightarrow \mathbb{R}_{+}^{*}$ of class $C^{1}$, nondecreasing and satisfying: $Q^{\prime} \leq C Q^{3 / 2}$ for some $C>0$ and $\int_{0}^{\infty} Q(t)^{-1 / 2} d t<\infty$. Then -1 is a small perturbation of $H$. In this form (and with slightly different assumptions on $V$ and $Q$ ), Corollary 9.1 was conjectured by Y. Pinchover [Pi2] who also noted that for $Q(t)=t^{2+\varepsilon}, \varepsilon>0$, the result is shown in [Mur, Theorem 5.8]. As in [Mur], our proof (cf. 9.4 below) uses a change of metric.

\section{3.}

The first step in the proof of Theorem 5 is the following elementary lemma. Let $\tilde{g}$ be a $C^{0}$-metric in $M$, conformal to $g$, i.e. $\tilde{g}_{x}(u, u)=K(x)^{2}$ $g_{x}(u, u), x \in M, u \in T_{x}^{1}(M)$, for some $C^{0}$ function $K$ in $M$. It is also assumed that $K \geq 1$. Denote $\tilde{d}$ the Riemannian distance in $M$ corresponding to $\tilde{g}$ and $\widetilde{\bar{B}}(m, r)$ (resp. $\bar{B}(m, r))$ the closed ball of center $m \in M$ and radius $r \geq 0$ in $(\widetilde{M}, \tilde{g})$ (resp. in $(M, g))$. We need a simple estimate of the derivative of the function

$$
F(R)=\sup \{\tilde{d}(O, x) ; x \in M, d(O, x) \leq R\}
$$

Clearly, $F(R)$ is the smallest $t \geq 0$ such that $\bar{B}(O, R) \subset \widetilde{\bar{B}}(O, t)$. The function $F$ is nondecreasing, continuous and even locally Lipschitz in $\mathbb{R}_{+}$. 
For if $C=\max \{K(x) ; x \in \bar{B}(O ; T)\}, T>0$, and if $0 \leq R \leq R^{\prime} \leq T$, we have for $x \in \bar{B}\left(O, R^{\prime}\right) \backslash B(0, R)$,

$$
\tilde{d}(O, x) \leq \tilde{d}\left(O, x^{\prime}\right)+\tilde{d}\left(x^{\prime}, x\right) \leq F(R)+C d\left(x^{\prime}, x\right) \leq F(R)+C\left(R^{\prime}-R\right)
$$

where $x^{\prime}$ is in the intersection of a $g$-geodesic segment $\overline{O x}$ with $\partial B(O, R)$. It follows that $F\left(R^{\prime}\right)-F(R) \leq C\left(R^{\prime}-R\right)$.

Using the same simple argument we may estimate the derivative $F^{\prime}(R)$. Let $S(O, R)$ denote the sphere $\{x ; d(O, x)=R\}$, let $\widetilde{S}(O, R)=\{x \in M$; $\tilde{d}(O, x)=R\}$ and let $\widetilde{S}_{0}(O, t) \subset \widetilde{S}(O, t)$ denote the boundary of $\widetilde{\widetilde{B}}(O, t)$ in $M$. By the assumptions on $M$ and $K, \widetilde{S}_{0}(O, R) \neq \emptyset$ but it may happen that the set $S(O, R) \cap \widetilde{S}(O, F(R))$ is empty.

LEMMA 9.2. Let $R>0$ and let $A(R)=S(O, R) \cap \widetilde{S}_{0}(O, F(R))$. Then, $F$ is constant in some interval $\left[R, R^{\prime}\left[, R^{\prime}>R\right.\right.$, if and only if $A(R)=\emptyset$. For almost all $R$ with $A(R) \neq \emptyset$,

$$
F^{\prime}(R) \leq \sup \{K(x) ; x \in A(R)\} .
$$

The first assertion is easily checked (by the continuity of $R \mapsto \bar{B}(O, R)$ and $t \mapsto \widetilde{\bar{B}}(O, t))$.

Fix $R>0$ with $A(R) \neq \emptyset$. For each $R^{\prime}>R$ pick $x \in \bar{B}\left(O, R^{\prime}\right) \backslash \bar{B}(O, R)$ with $F\left(R^{\prime}\right)=\tilde{d}(O, x)$, then as before

$$
\tilde{d}(O, x)-F(R) \leq \tilde{d}(O, x)-\tilde{d}(O, y)
$$

where $y$ is chosen in the intersection of a minimizing $g$-geodesic $\gamma$ joining $O$ to $x$ with the sphere $S(O, R)$. Thus $F\left(R^{\prime}\right)-F(R) \leq \tilde{d}(x, y) \leq\left(K(y)+\varepsilon\left(R^{\prime}-\right.\right.$ $R))\left(R^{\prime}-R\right)$ where $\varepsilon(\tau)=\sup \left\{\left|K(z)-K\left(z^{\prime}\right)\right| ; d\left(z, z^{\prime}\right) \leq \tau, d(O, z) \leq R\right\}$. Whence

$$
\limsup _{R^{\prime} \downarrow R} \frac{F\left(R^{\prime}\right)-F(R)}{R^{\prime}-R} \leq \sup \{K(z) ; z \in A(R)\}
$$

and the lemma follows.

\subsection{Proof of Theorem 5}

Introduce the Riemannian metric $\tilde{g}_{x}=V_{0}(x) g_{x}, x \in M$, in $M$. It is easily checked that $(M, \tilde{g})$ is a complete Riemannian manifold with a $C^{1}$ metric (each $\tilde{g}$-ball, being contained in the $g$-ball with same radius and center, is relatively compact in $M)$. Moreover, the standard bounded geometry assumptions ([An4, Section 1.1]) hold for $(M, \tilde{g})$ by the following elementary lemma. Recall $C_{0}$ is a constant $\geq 1$ such that $\left|\nabla V_{0}\right| \leq C_{0} V_{0}^{3 / 2}$. 
LEMMA 9.3. If $m_{0}$ and $m$ are such that $d\left(m_{0}, m\right) \leq \frac{1}{8 C_{0}} V_{0}\left(m_{0}\right)^{-1 / 2}$ then

$$
\frac{1}{2} V_{0}\left(m_{0}\right) \leq V_{0}(m) \leq 2 V_{0}\left(m_{0}\right)
$$

Proof. If we have $V_{0}(m)>2 V_{0}\left(m_{0}\right)$, then we may find on some chosen minimizing geodesic segment $m_{0} m$ a point $m^{\prime}$ nearest to $m_{0}$ such that $V_{0}\left(m^{\prime}\right)=2 V_{0}\left(m_{0}\right)$. Then, $V_{0}\left(m_{0}\right)=V_{0}\left(m^{\prime}\right)-V_{0}\left(m_{0}\right) \leq C_{0} d\left(m^{\prime}, m_{0}\right)$ $\sup _{\left[m_{0}, m^{\prime}\right]} V_{0}(z)^{3 / 2}=2^{3 / 2} C_{0} d\left(m^{\prime}, m_{0}\right) V_{0}\left(m_{0}\right)^{3 / 2}$.

Thus, $1 / \sqrt{V_{0}\left(m_{0}\right)} \leq 2^{3 / 2} C_{0} d\left(m_{0}, m\right)$ which contradicts the assumption. This proves the second inequality in the lemma. The first one then follows:

$$
\begin{aligned}
\left|V_{0}(m)-V_{0}\left(m_{0}\right)\right| & \leq C_{0} d\left(m_{0}, m\right) \sup _{z \in\left[m_{0}, m\right]}\left|V_{0}(z)\right|^{3 / 2} \\
& \leq \frac{1}{8} 2^{3 / 2} V_{0}\left(m_{0}\right) \leq \frac{1}{2} V_{0}\left(m_{0}\right)
\end{aligned}
$$

It follows from Lemma 9.3, that $\widetilde{B}\left(m_{0}, r_{0}^{\prime}\right)$, the $\tilde{g}$ ball of center $m_{0}$ and radius $r_{0}^{\prime}=r_{0} / 17 C_{0}$, is contained in the $g$-ball $B\left(m_{0}, r\left(m_{0}\right)\right), r\left(m_{0}\right)=$ $\left(8 C_{0} V_{0}\left(m_{0}\right)^{1 / 2}\right)^{-1} r_{0} \leq\left(8 C_{0} V_{0}\left(m_{0}\right)^{1 / 2}\right)^{-1}$. On taking $\tilde{\chi}_{m_{0}}(z)=\sqrt{V_{0}\left(m_{0}\right)}$ $\chi_{m_{0}}(z)$ (see 9.1$)$ we obtain a chart $\tilde{\chi}_{m_{0}}: \widetilde{B}\left(m_{0}, r_{0}^{\prime}\right) \rightarrow \mathbb{R}^{n}$ which is $c_{1}$ bilipschitz w.r. to $\tilde{g}$ with $c_{1}=\sqrt{2} c_{0}$. So $(M, \tilde{g})$ has bounded geometry as announced.

The gradient operator $\widetilde{\nabla}$ with respect to $\tilde{g}$ is given by the formula $\widetilde{\nabla} .=$ $V_{0}(x)^{-1} \nabla$., and for the divergence operator we have $\widetilde{\operatorname{div}}()=.V_{0}(x)^{-n / 2}$ $\operatorname{div}\left(V_{0}^{n / 2}\right.$.) (where $\nabla$ and $\operatorname{div}$ are related to $(M, g)$ and $\left.n=\operatorname{dim}(M)\right)$. Thus, for $j=1,2$,

$$
\begin{aligned}
L_{j}(u) & =V_{0}^{n / 2} \widetilde{\operatorname{div}}\left(V_{0}^{1-n / 2} \mathcal{A}_{j} \widetilde{\nabla} u\right)+B_{j} \sim \widetilde{\nabla} u-V_{j} u \\
& =V_{0}\left\{\widetilde{\operatorname{div}}\left(\mathcal{A}_{j} \widetilde{\nabla} u\right)+\left[\left(1-\frac{n}{2}\right) V_{0}^{-1} \mathcal{A}_{j}^{*}\left(\widetilde{\nabla} V_{0}\right)+\frac{1}{V_{0}} B_{j}\right] \sim \widetilde{\nabla} u-\frac{V_{j}}{V_{0}} u\right\}
\end{aligned}
$$

where . refers to the inner product with respect to $\tilde{g}$.

Set for $j=1,2, \widetilde{L}_{j}:=\frac{1}{V_{0}} L_{j}=\widetilde{\operatorname{div}}\left(\mathcal{A}_{j} \widetilde{\nabla}.\right)+\left[(1-n / 2) V_{0}^{-1} \mathcal{A}_{j}^{*}\left(\widetilde{\nabla} V_{0}\right)+\right.$ $\left.\frac{1}{V_{0}} B_{j}\right] \sim \widetilde{\nabla} .-\frac{V_{j}}{V_{0}}$. It is easily checked that the elliptic operator $\widetilde{L}_{j}$ is adapted on $\widetilde{M}=(M, \tilde{g})$ (it has a bounded drift in $(M, \tilde{g})$ and a bounded zero order coefficient, so in the notations of [An4], $\widetilde{L}_{j} \in \mathcal{D}_{\widetilde{M}}(\theta, \infty)$ for $\theta$ chosen large enough). Moreover, $\widetilde{L}_{j}+c$ is transient in $M \backslash \bar{B}(O, R)$ for $c<1 / C_{1}$ and $R$ 
large, and $\widetilde{L}_{j}$ is transient in $M$, thus $\widetilde{L}_{j}$ is weakly coercive in $M$ (see [An4, Corollary 1.1], and its proof for the simple argument).

We may then apply the main result in [An4] and compare the Green functions $\widetilde{G}_{1}$ and $\widetilde{G}_{2}$ for $\widetilde{L}_{1}$ and $\widetilde{L}_{2}$ over $(\widetilde{M}, \tilde{g})$, since for $\widetilde{B}_{j}=V_{0}^{-1}\left[B_{j}+\right.$ $\left.(1-n / 2) \mathcal{A}_{j}^{*}\left(\widetilde{\nabla} V_{0}\right)\right]$ and

$$
\begin{aligned}
\Psi(t)=\sup \left\{\| \mathcal{A}_{1}(x)-\right. & \mathcal{A}_{2}(x)\left\|_{\tilde{g}}+\right\| \widetilde{B}_{1}(x)-\widetilde{B}_{2}(x) \|_{\tilde{g}} \\
& \left.+\frac{\left|V_{1}(x)-V_{2}(x)\right|}{V_{0}(x)} ; \tilde{d}(0, x) \geq t\right\}
\end{aligned}
$$

we have $\Psi(F(r)) \leq\left(C_{0} n / 2\right) \Phi(r)$, and by Lemma 9.2

$$
\begin{aligned}
\int_{0}^{\infty} \Psi(t) d t & =\int_{0}^{\infty} \Psi(F(R)) F^{\prime}(R) d R \\
& \leq C_{0} \frac{n}{2} \int_{0}^{\infty} \Phi(R) \sup \left\{\sqrt{V_{0}(x)} ; d(0, x)=R\right\} d R<\infty
\end{aligned}
$$

So, by $\left[\right.$ An4, Theorem 1] there is a $C \geq 1$ such that $C^{-1} \widetilde{G}_{1}(x, y) \leq$ $\widetilde{G}_{2}(x, y) \leq C \widetilde{G}_{1}(x, y)$ if $\tilde{d}(x, y) \geq 1$. Since $\widetilde{\widetilde{G}}_{j}(x, y)=G_{j}(x, y) V_{0}(y)^{1-n / 2}$ (see definition of Green's function in $\S 1$ ) the result follows.

\subsection{Proof of Corollary 9.1}

Under the assumptions of Corollary 9.1, the proof above (for $L_{2}=$ $L_{1}-1$ ) and [An4] Section 6 show that $-1 / V_{0}$ is a small perturbation of $\widetilde{L}_{1}$ in $(M, \tilde{g})$, that is

$\sup \left\{\left[\int_{\tilde{d}(0, z) \geq T} \widetilde{G}_{1}(x, z) \frac{1}{V_{0}(z)} \widetilde{G}_{1}(z, y) d \tilde{\sigma}(z)\right] / \widetilde{G}_{1}(x, y) ; x, y \notin \widetilde{B}(0, T)\right\} \rightarrow 0$

as $T \rightarrow \infty$. Since $\widetilde{G}_{1}(x, y)=G_{1}(x, y) V_{0}(y)^{1-n / 2}$,

$$
\begin{aligned}
& \int_{\tilde{d}(0, z) \geq T} \widetilde{G}_{1}(x, z) \frac{1}{V_{0}(z)} \widetilde{G}_{1}(z, y) d \tilde{\sigma}(z) \\
&=V_{0}(y)^{1-n / 2}\left[\int_{\tilde{d}(0, z) \geq T} G_{1}(x, z) G_{1}(z, y) V_{0}(z)^{-n / 2} d \tilde{\sigma}(z)\right]
\end{aligned}
$$

or

$$
\begin{aligned}
& \frac{1}{\widetilde{G}_{1}(x, y)} \int_{\tilde{d}(0, z) \geq T} \widetilde{G}_{1}(x, z) \frac{1}{V_{0}(z)} \widetilde{G}_{1}(z, y) d \tilde{\sigma}(z) \\
& =\frac{1}{G_{1}(x, y)} \int_{\tilde{d}(0, z) \geq T} G_{1}(x, z) G_{1}(z, y) d \sigma(z) .
\end{aligned}
$$


So (9.1) means that -1 is a small perturbation of $L_{1}$ in $M$.

\section{§10. Proof of Theorem $2^{\prime}$}

We start with a discrete setting counter-example. A "continuous setting" counter part is briefly described afterwards.

\subsection{A discrete setting example with bounded geometry and bounded drift}

By a segment of length $\ell$ (where $\ell \in \mathbb{N}^{*}$ ) we shall mean a graph $S$ of the form $S=\left\{A_{0}, A_{1}, \ldots, A_{\ell}\right\}$ with nearest neighbor relations: $A_{j} \sim A_{k}$ if and only if $|j-k|=1,0 \leq j, k \leq \ell$. We shall also denote $S=\left[A_{0}, A_{\ell}\right]$. Notice that for the random walk in $S$ with the transition function $p\left(A_{j},.\right)=$ $\frac{2}{3} \delta_{A_{j-1}}+\frac{1}{3} \delta_{A_{j+1}}, 1 \leq j \leq \ell-1, p\left(A_{0}, A_{0}\right)=p\left(A_{\ell}, A_{\ell}\right)=1$, the probability, starting from $A_{k}$ to hit $A_{\ell}$ before hitting $A_{0}$ is smaller than $2^{k-\ell}$ and hence small for $\ell-k$ large.

Consider the sequence $P_{j}=\left(2^{j}, 0,0\right), j \geq 1$, in $\mathbb{Z}^{3}$; by the Wiener type criterion $([\mathrm{I}-\mathrm{M}])$, the set $\left\{P_{j} ; j \geq 0\right\}$ is thin at infinity for the standard random walk in $\mathbb{Z}^{3}$. Construct a graph $X$ by first glueing to $\mathbb{Z}^{3}$ (with its standard graph structure of constant valence 6) at each $P_{j}$ a segment $\left[P_{j}, Q_{j}\right]=\left\{P_{j}^{0}, \ldots, P_{j}^{\ell_{j}}\right\}$ of length $\ell_{j} \geq 10$ to be chosen later (so $P_{j}^{0}=P_{j}$, $\left.P_{j}^{\ell_{j}}=Q_{j}\right)$, and then segments $\left[Q_{j}, Q_{j+1}\right]=\left\{Q_{j}^{0}, Q_{j}^{1}, \ldots, Q_{j}^{L_{j}}\right\}$ of length $L_{j} \geq 10$ (so $Q_{j}^{L_{j}}=Q_{j+1}$ ). To be more specific, each $P_{j}=P_{j}^{0}$ has seven neighbors: the six standard neighbors in $\mathbb{Z}^{3}$ and $P_{j}^{1}$, the vertex $Q_{1}$ has two neighbors: $Q_{1}^{1}$ and $P_{1}^{\ell_{1}-1}$, and $Q_{j}, j \geq 2$, has three neighbors: $Q_{j}^{1}$, $Q_{j-1}^{L_{j-1}-1}$ and $P_{j}^{\ell_{j}-1}$. Let $\Sigma_{j}$ denote the union of all segments $\left[P_{k}, Q_{k}\right]$, $\left[Q_{k}, Q_{k+1}\right]$, with $1 \leq k \leq j-1$ and set $B_{j}=\left\{z \in \mathbb{Z}^{3} ;|z| \leq 2^{j+2}\right\} \cup \Sigma_{j+2}$, $B_{j}^{\prime}=\left\{z \in \mathbb{Z}^{3} ;|z| \leq 2^{j+1 / 2}\right\} \cup \Sigma_{j+1}$ where $|z|=\max _{1 \leq i \leq 3}\left|z_{i}\right|$ for $z \in \mathbb{Z}^{3}$.

Consider then the (adapted) random walk on $X$ with the transition probability measures $p(m,),. m \in X$, such that (we denote $\sim$ the nearest neighbor relation in $X)$ :

(i) $p(m, y) \neq 0$ if and only if $y \in V_{m}=\{z \in X ; m \sim z\}$,

(ii) if $m \in X$ is not interior to one of the added segments, $p(m,)=$. $\left|V_{m}\right|^{-1} \sum_{y \sim x} \delta_{y}$

(iii) $p\left(P_{j}^{k},.\right)=\frac{1}{3} \delta_{P_{j}^{k-1}}+\frac{2}{3} \delta_{P_{j}^{k+1}}$ for $1 \leq k \leq \ell_{j}-1$,

(iv) $p\left(Q_{j}^{k},.\right)=\frac{2}{3} \delta_{Q_{j}^{k-1}}+\frac{1}{3} \delta_{Q_{j}^{k+1}}$ for $1 \leq k \leq L_{j}-1$. 
Thus on the interior of each of the added segments, the random walk is a standard random walk with a constant drift. It is then easy to see that

(a) the pair $(X, p)$ is transient $\left(\mathbb{Z}^{3}\right.$ being transient and $\left\{P_{j} ; j \geq 1\right\}$ thin at infinity in $\mathbb{Z}^{3}$ ),

(b) if the length $\ell_{j}$ grow sufficiently fast (independently of the choice of the lengths $L_{j}$ ) then the hitting probability

$$
P\left\{\exists n \geq 1: Z_{n}=Q_{1} \text { and } Z_{s} \notin \mathbb{Z}^{3}, \forall s \leq n \mid Z_{0}=m\right\}
$$

is at least $1 / 2$ for every starting point $m \in \mathcal{R}=\bigcup_{j \geq 1}\left[Q_{j}, Q_{j+1}\right]$,

(c) fixing $\left\{\ell_{j}\right\}$ so that (b) is satisfied, one may then choose successively the $L_{k}$ so large that the probability, starting from $m=Q_{k}^{s}, 0 \leq s \leq \frac{3}{4} L_{k}$, to hit $Q_{k+1}$ before $Q_{k}$ is smaller than any given $\varepsilon_{k}$. In particular, if $L_{k}$ is sufficiently large, then for every positive $p$-harmonic function in $B_{k+1}$, we have (for a constant $C$ independent of $k$ )

$$
\sup _{x \in \partial B_{k}^{\prime}} u(x) \leq C u\left(-2^{k}, 0,0\right) \text {. }
$$

The proof is similar to the proof of (5.1) and in fact easier since for $A_{k}=$ $\left\{z \in \mathbb{Z}^{3} ;|z|=\sqrt{2} 2^{k}\right\}$ we may easily obtain from the available uniform Harnack inequalities w. r. to the standard random walk (Remark 2 below) the estimate $\sup _{x \in A_{k}} u(x) \leq C u\left(-2^{k}, 0,0\right)$. So substitutes to Proposition 4.1 or to the killing procedure in Section 5 are not needed.

Arguing as in Section 3, it is seen that the Martin boundary of the random walk constructed above is trivial, i.e. positive $p$-harmonic functions in $X$ are constants. On the other hand, by (b) above, the Green function $G\left(., Q_{1}\right)$ is larger than a constant on the ray $\mathcal{R}=\bigcup_{j \geq 1}\left[Q_{j}, Q_{j+1}\right]$. Finally we have a graph with bounded geometry (i.e. the number of neighbors of each vertex $m \in X$ is bounded by a constant) with an adapted transient random walk whose boundary is trivial but whose Green's function $G_{O}$ does not vanish at infinity.

Remarks. 1. Let us mention and prove more explicitly the analogue of Lemma 5.1. If $\psi$ is harmonic measure of the point $Q_{j+1}$ in $B_{j}^{\prime}$, we have (using a "path" $\left[Q_{j}, P_{j}\right] \cup \gamma$ joining $Q_{j}$ to $\partial B_{j}^{\prime}$ in $X$ with $\gamma \subset \mathbb{Z}^{3}$ ) that $\psi\left(Q_{j}\right) \leq\left(1-c_{j}\right) \psi\left(Q_{j}^{1}\right)$ where $c_{j}>0$ depends only on $j$ and $\ell_{j}$. Writing $\psi=\varphi+H_{\psi}$ in $\left[Q_{j}, Q_{j+1}\right]$ where $\varphi$ is the harmonic measure of $Q_{j+1}$ in 
$\left[Q_{j}, Q_{j+1}\right]$, we have

$$
\begin{array}{r}
\psi\left(Q_{j}^{k}\right)=\varphi\left(Q_{j}^{k}\right)+H \psi\left(Q_{j}^{k}\right) \leq \varphi\left(Q_{j}^{k}\right)+\psi\left(Q_{j}\right) \leq \varphi\left(Q_{j}^{k}\right)+\left(1-c_{j}\right) \psi\left(Q_{j}^{k}\right) \\
1 \leq k \leq L_{j}
\end{array}
$$

(using maximum principle two times) whence $\psi\left(Q_{j}^{k}\right) \leq c_{j}^{-1} \varphi\left(Q_{j}^{k}\right) \leq c_{j}^{-1}$ $2^{k-L_{j}}$. Thus for any given $\varepsilon>0$, we have as before $\psi\left(Q_{j}^{k}\right) \leq \varepsilon$ for all $k \leq \frac{3}{4} L_{j}$ as soon as $L_{j}$ is large enough.

2. The (well-known) Harnack inequalities for the standard random walk $\zeta=\left\{\zeta_{n}\right\}$ in $\mathbb{Z}^{3}$ (see [Duf]) say that there is a $c_{0} \geq 1$ such that for $R \geq 1$, and $u$ non-negative and $\zeta$-harmonic in $\omega_{R}=\left\{z \in \mathbb{Z}^{3} ;|z| \leq R\right\}$ one has $c_{0}^{-1} u(0) \leq u(x) \leq c_{0} u(0)$ for all $x \in \omega_{R / 2}$.

\subsection{A Riemannian manifold example}

It is easy to produce a similar example with a complete Riemannian manifold $M$ of bounded geometry and an elliptic operator of the form $\mathcal{L}=$ $\Delta+V . \nabla$ where $V$ is a bounded drift in $M$. Associate to each abstract edge from $X$ a (flat) cylinder of length and radius equal to one, each boundary circle corresponding to one of the vertices defining the edge. Associate also to each vertex of valence $\geq 3$ a sphere of radius ten. Glue together in the natural way the cylinders' boundary circles corresponding to the same vertex of valence 2 , and for each vertex of valence $\geq 3$, attach (smoothly and in a uniform way) to the corresponding sphere the boundary circles associated to this vertex. Equip the resulting Riemannian manifold with the operator $\mathcal{L}=\Delta+V . \nabla$ where $V$ is a unit vector field parallel to the axis on the cylinders with at least one corresponding vertex outside $\mathbb{Z}^{3}$ (and same orientation as the discrete drift in the preceding construction) and $V$ vanishes elsewhere. Then again, by arguments similar to 10.1 and $\S 5$, it may be shown that if the $\ell_{j}$ are first chosen long enough and then the $L_{j}$ taken very large, the Martin boundary of $(M, \mathcal{L})$ is trivial but the Green's function of $\mathcal{L}$ does not vanish at infinity. Clearly, $M$ has bounded sectional curvatures and injectivity radius bounded from below. Also $V$ is bounded (and can obviously be made smooth by a slight change in the construction).

\section{§11. Appendix. Proof of Remarks 1.2}

Part A. It is immediate that (b) implies (c) and easy to check that (c) implies (b) for $x$ and $y$ in $M \backslash\{O\}$ and $f(x)=G(O, x) / G(x, O)$; the complete property (b) follows. That (b) implies (a) is clear once noted 
that if $u \in C_{0}^{2}(M)$ then $u=G(\varphi)$ with $\varphi=-\mathcal{L}(u)$ (the $\mathcal{L}$-subharmonic function $|u-G(\varphi)|$ is majorized near infinity by the potential $G(|\varphi|)$ and thus vanishes).

It remains to prove that (a) implies (b). Fix $p \in(N, \infty), N=\operatorname{dim}(M)$. Assuming (a), a simple approximation argument shows that (a) holds for $u$ and $v$ compactly supported and of class $W_{\mathrm{loc}}^{2, p}$. Let $\varphi$ and $\psi$ be nonegative compactly supported functions in $L^{p}(M)$. Then, by standard $L^{p}$ interior estimates, $G(\varphi)$ is in $W_{\text {loc }}^{2, p}(M)$. If $K$ is a large compact subset in $M$, if $K^{\prime}$ is a compact neighborhood of $K$ and if $\alpha: M \rightarrow[0,1]$ is smooth, vanishes in $K$ and is equal to 1 in $M \backslash K^{\prime}, w=\alpha G(\varphi)$ is in $W^{2, p}$ and $w=G(\tilde{\varphi})$, if $\tilde{\varphi}=-\mathcal{L}(w)$. By a general property of réduites, $G\left(\tilde{\varphi}_{+}\right)-R(w)$ is a $\mathcal{L}$ potential (ref. [Mok, pp. 220-221]), so $R(w)=G\left(\varphi_{1}\right)$ where $\varphi_{1} \in L_{+}^{p}(M)$ and vanishes outside $K^{\prime} \backslash K$. Starting from $\psi$ and compacts subsets $L \subset L^{\prime}$ -instead of $\varphi, K$ and $K^{\prime}$ - there is a similar function $\psi_{1} \in L^{p}(M)$. We may now apply the identity in (a) to $u=G(\varphi)-G\left(\varphi_{1}\right)$ and $v=G(\psi)-G\left(\psi_{1}\right)$.

Fixing $K$ and taking $L$ large we have $\int\left(\varphi-\varphi_{1}\right) G\left(\psi-\psi_{1}\right) f d \sigma=$ $\int \psi G\left(\varphi-\varphi_{1}\right) f d \sigma$. Now as $L$ tends to $M, G\left(\psi_{1}\right)$ decreases to zero (by the definitions of potentials). So, $\int\left(\varphi-\varphi_{1}\right) G(\psi) f d \sigma=\int \psi G\left(\varphi-\varphi_{1}\right) f d \sigma$. Let then $K$ grow to $M$ : from $\int \psi G\left(\varphi-\varphi_{1}\right) f d \sigma \leq \int \varphi G(\psi) f d \sigma$ we get $\int \psi G(\varphi) f d \sigma \leq \int \varphi G(\psi) f d \sigma$. Exchanging $\varphi$ and $\psi$ we finally obtain

$$
\int \psi G(\varphi) f d \sigma=\int \varphi G(\psi) f d \sigma,
$$

for all compactly supported and non-negative $\varphi, \psi$ in $L^{p}(M)$. (b) follows with $f=h$.

Part B. Assume that $\mathcal{L} \equiv \Delta .+D . \nabla .+\gamma$. and that $\theta$ is symmetric. Writing (a) above, we first see by taking $v=1$ on the support of $u$ that $\Delta f=\operatorname{div}(f D)$ in the distribution sense. Taking $u$ and $v$ arbitrary, we obtain that in the distribution's sense and for all $u \in C_{0}^{\infty}(M)$ :

$$
f[\Delta u+D . \nabla u]=\Delta(f u)-\operatorname{div}(u f D) .
$$

Now, $\Delta(f u)-\operatorname{div}(u f D)=u[\Delta(f)-\operatorname{div}(f D)]+2 \nabla u . \nabla f+(\Delta u) f-f D . \nabla u$. Thus, we get

$$
2 f D . \nabla u=2 \nabla u . \nabla f
$$

for all $u \in C_{0}^{\infty}(M)$, in the distribution sense. So, $D=\nabla \log (f) \sigma$-a.e. (and $f$ is locally Lipschitz since $D$ is locally bounded). The reverse implication ( $D$ is a gradient implies the symmetry of $\theta$ ) is left to the reader. 
Remark 11.1. It follows from Remarks 1.2 that the symmetry of the $\theta$ kernel is a "local" property (i.e. it holds for $(M, \mathcal{L})$ iff it holds in $\left(U_{j}, \mathcal{L}_{\mid U_{j}}\right)$ for some open cover $\left\{U_{j}\right\}$ of $\left.M\right)$ if $M$ is simply connected-or more generally if $H^{1}(M, \mathbb{R})=0-$, but not in general.

\section{REFERENCES}

[An1] A. Ancona, Une propriété de la compactification de Martin d'un domaine euclidien, Ann. Inst. Fourier (Grenoble), 29, 4 (1979), 71-90.

[An2] — Negatively curved manifolds, elliptic operators and the Martin boundary, Ann. of Math., 125 (1987), 495-536.

[An3] Théorie du Potentiel sur les graphes et les variétés, Ecole d'été de Probabilités de Saint-Flour XVIII - 1988, Lecture Notes in Math. 1427, Springer-Verlag (1990), pp. 5-112.

[An4] - First eigenvalues and comparison of Green's functions for elliptic operators on manifolds or domains, J. d'Analyse Math., 72 (1997), 45-92.

[An5] — Green's functions, generalized first eigenvalues and perturbations of diffusions or Markov chains, Random walks and discrete Potential Theory (M. Picardello and W. Woess, eds.), Symposia Mathematica XXXIX, Cambridge Univ. Press (1999), pp. 1-25.

[Aro] N. Aronszajn, A unique continuation theorem for solutions of elliptic partial differential equations or inequalities of second order, J. Math. Pures Appl., (9) 36 (1957), 235-249.

[Bre] M. Brelot, Axiomatique des fonctions harmoniques, Les Presses de l'Université de Montréal, 1969.

[Cho] G. Choquet, Diamètre tranfini et comparaison de diverses capacités, Séminaire de Théorie du Potentiel, faculté des Sciences de Paris, année 1958-59 (1960), exposé $\mathrm{n}^{\circ} 4$.

[Duf] R. J. Duffin, Discrete potential theory, Duke Math. J., 20 (1953), 233-251.

[Ev] G. C. Evans, Potentials and positively infinite singularities of harmonic functions, Monatsch. Math. Phys., 43 (1936), 419-424.

[Fek] M. Fekete, Über die Verteilung des Wurzeln bei geweissen algebraischen Gleichungen mit ganzzahligen Koeffizienten, Math. Zeitschr., 17 (1923), 228-249.

[Her] R.-M. Hervé, Recherche sur la théorie axiomatique des fonctions surharmoniques et du Potentiel, Ann. Inst. Fourier, XII (1962), 415-471.

[H-H] M. Hervé and R.-M. Hervé, Les fonctions surharmoniques associées à un opérateur elliptique du second ordre à coefficients discontinus, Ann. Inst. Fourier, XIX (1969), 305-359.

[Hör] L. Hörmander, The analysis of linear partial differential operators III, Grundlehren der Mathematischen Wissenschaften, Springer-Verlag, Berlin, 1985.

[I-M] K. Itô and H. P. McKean, Potentials and the random walk, Illinois J. Math., 4 (1960), 119-132. 
[K-V] N. J. Kalton, I. E. Verbitsky, Nonlinear equations and weighted norm inequalities, Trans. Amer. Math. Soc., 351 (9) (1999), 3441-3497.

[Mok] G. Mokobodzki, Cônes de potentiels et noyaux subordonnés, Potential Theory (C.I.M.E., I Ciclo, Stresa, 1969), Edizioni Cremonese, Roma (1970), pp. 207-248.

$[\mathrm{Mu}]$ M. Murata, Structure of positive solutions to $(-\Delta+V) u=0$ in $R^{n}$, Duke Math. J., 53 (1986), 869-943.

[Naï] L. Naïm, Sur le rôle de la frontière de R. S. Martin dans la théorie du Potentiel, Annales de l'institut Fourier, 7 (1957), 183-281.

[Ni1] N. Ninomiya, Sur le principe de continuité dans la théorie du Potentiel, J. Inst. Polytech. Osaka City Univ. Ser. A. 8 (1957), 51-56.

[Ni2] — A note on transfinite diameter, Kōdai Math. Sem. rep., 27, 3 (March, 1976), 300-307.

[Owh] H. Owhadi, Private communications (1999).

[Pi1] Y. Pinchover, Criticality and ground states for second-order elliptic equations, J. Differential Equations, 80 (1989), 237-250.

[Pi2] _ Private communications (September, 1998 and March, 1999).

[Pi3] Maximum and anti-maximum principles and eigenfunctions estimates via perturbation theory of positive solutions of elliptic equations, Math. Ann., 314 (1999), 555-590.

[Pi4] _ On the maximum and anti-maximum principles, Differential Equations and Mathematical Physics (R. Weikard and G. Weinstein, eds.), Symposia Mathematica XXXIX, International Press, Cambridge, MA (to appear).

[Sta] G. Stampacchia, Le problème de Dirichlet pour les équations elliptiques du second ordre à coefficients discontinus, Ann. Inst. Fourier, 15 (1) (1965), 189-258.

Département de Mathématiques

Bâtiment 425

Université Paris-Sud

91405 Orsay

France

ancona@matups . math. u-psud.fr 